\title{
Gerber-Shiu Analysis of a Risk Model with Capital Injections
}

\author{
David C M Dickson and Marjan Qazvini
}

\begin{abstract}
We consider the risk model with capital injections studied by Nie et al. (2011, 2015). We construct a Gerber-Shiu function and show that whilst this tool is not efficient for finding the ultimate ruin probability, it provides an effective way of studying ruin related quantities in finite time. In particular, we find a general expression for the joint distribution of the time of ruin and the number of claims until ruin, and find an extension of Prabhu's (1961) formula for the finite time survival probability in the classical risk model. We illustrate our results in the case of exponentially distributed claims and obtain some interesting identities. In particular, we generalise results from the classical risk model and prove the identity of two known formulae for that model.
\end{abstract}

Keywords: Capital injections; Gerber-Shiu function; ruin probability; finite time ruin; number of claims until ruin; exponential claims

\section{Introduction}

In this paper we analyse some ruin related quantities in the risk model with capital injections which was introduced by Nie et al. (2011). In this model the insurer's surplus starts at a level $u \geq k$, where $k>0$ is a fixed constant. On any occasion that the surplus falls between the levels 0 and $k$ from above $k$, a capital injection restores the surplus level to $k$. If the surplus falls below 0 from a level above $k$, ruin occurs.

Nie et al. (2011) explain how the capital injections can be provided by reinsurance and how an insurer can both reduce its ultimate ruin probability by effecting such reinsurance and release capital to other parts of its business. In a subsequent paper, Nie et al. (2015) consider finite time ruin probabilities under this framework. They obtain a general expression for the finite time ruin probability which can be evaluated in certain cases, and they find an expression for the density of the time of ruin when the underlying risk process is a Sparre Andersen process with Erlang claim arrivals and exponentially distributed claim amounts.

In this paper we take a different approach, with the aim of obtaining more general results than those obtained in Nie et al. (2015). We perform analysis based on the Gerber-Shiu function introduced in Landriault et al. (2011) which extends the original Gerber-Shiu function (Gerber and Shiu (1998)) by incorporating the number of claims until ruin into the analysis. We obtain some general results relating to finite time ruin problems on the assumption that the underlying risk process is a classical risk process, and we obtain specific results in the case of exponentially distributed claim amounts. GerberShiu functions have been studied for a variety of risk models, for example by Gerber and 
Shiu (2005) for a Sparre Andersen model, by Li and Lu (2008) for a Markov-modulated risk model, and by Lin et al. (2003) for a classical risk model with dividends. There seems to be little new to say about Gerber-Shiu functions. A contribution in this paper that differs from previous analyses is that we are interested in a Gerber-Shiu function defined for initial surplus $u \geq k$, meaning that our analysis requires construction of a function when $u=k$. However, the bigger contribution here is a surprising result that our analysis yields, namely that an extension of Prabhu's (1961) formula for the finite time ruin probability for the classical risk model exists for the risk model with capital injections.

This paper is organised as follows. In Section 2 we give details of our model, introduce some notation and motivate our study. Then, in Section 3, we define our Gerber-Shiu function and obtain an expression for its Laplace transform and for the Gerber-Shiu function itself when the initial surplus is $k$. We show in Section 4 how a formula for the ultimate ruin probability can be found from our Gerber-Shiu function, then in Section 5 we consider the joint distribution of the time of ruin and the number of claims until ruin. In particular, we obtain a version of Prabhu's (1961) formula for the density of the time of ruin and we obtain a more compact solution for this density when claim amounts are exponentially distributed compared with the formula obtained by Nie et al. (2015). Finally, in Section 6 we consider an alternative view of our Gerber-Shiu function and obtain a neat expression for the probability function of the number of claims until ruin, and show how the ultimate ruin probability can be found from this probability function.

\section{Model, notation and motivation}

The underlying model for our study is the classical risk model which we now describe. The surplus process in the classical risk model is $\{U(t)\}_{t \geq 0}$ where $U(t)=u+c t-S(t)$, with $u$ being the initial surplus, and $c$ being the rate of premium income per unit time (assumed to be received continuously). Further $S(t)=\sum_{i=1}^{N(t)} X_{i}$ where $\{N(t)\}_{t \geq 0}$ is a Poisson process with Poisson parameter $\lambda$ and $\left\{X_{i}\right\}_{i=1}^{\infty}$ is a sequence of independent and identically distributed random variables, where $X_{i}$ represents the amount of the $i$ th claim. Let $F=1-\bar{F}$ be the distribution function of $X_{1}$, with $F(0)=0$, and density function $f$. Let $\mathcal{G}(x, t)=\operatorname{Pr}(S(t) \leq x)$ with density function

$$
g(x, t)=\frac{d}{d x} \mathcal{G}(x, t)=\sum_{n=1}^{\infty} e^{-\lambda t} \frac{(\lambda t)^{n}}{n !} f^{n *}(x)
$$

for $x>0$, where $f^{n *}$ denotes the $n$-fold convolution of $f$ with itself.

Throughout we assume that the premium is calculated as $c=(1+\theta) \lambda E\left(X_{1}\right)$ where $\theta>0$ is the premium loading factor.

The time of ruin is denoted by $T$ and is defined as $T=\inf \{t: U(t)<0 \mid U(0)=u\}$ with $T=\infty$ if $U(t) \geq 0$ for all $t>0$. The ultimate ruin probability is defined as $\psi(u)=\operatorname{Pr}(T<\infty \mid U(0)=u)=1-\phi(u)$; the finite time ruin probability is

$$
\operatorname{Pr}(T \leq \tau \mid U(0)=u)=\int_{0}^{\tau} w(u, t) d t
$$

where $w(u, t)$ is the defective density of the time of ruin. The deficit at ruin is denoted by $|U(T)|$ and the probability and severity of ruin function is

$$
G(u, x)=\operatorname{Pr}(T<\infty \text { and }|U(T)| \leq x \mid U(0)=u)=\int_{0}^{\infty} \int_{0}^{x} w(u, y, t) d y d t
$$


where $w(u, y, t)$ is the defective joint density of $|U(T)|$ and $T$. It is well known, e.g. Gerber et al. (1987), that

$$
g(0, x)=\frac{d}{d x} G(0, x)=\frac{\lambda}{c} \bar{F}(x) .
$$

Our risk model of interest is a classical risk model modified by capital injections. Specifically, starting from initial surplus $u \geq k>0$, if the surplus process falls between 0 and $k$ a capital injection restores the surplus level to $k$, and ruin occurs only if the surplus process falls below 0 from a level above $k$; Figure 5.3 shows a realisation of the surplus process with capital injections for which ruin occurs. For the surplus process with capital injections we use the same notation as for the classical risk model, but with a subscript $k$, so that, for example, $T_{k}$ denotes the time of ruin and $\psi_{k}(u)$ denotes the ultimate ruin probability from initial surplus $u$.

Throughout this paper the Laplace transform of a function $\alpha$ will be denoted by $\tilde{\alpha}$ so that $\tilde{\alpha}(s)=\int_{0}^{\infty} e^{-s x} \alpha(x) d x$. An important Laplace transform is that of $S(t)$ which is given by

$$
\int_{0}^{\infty} e^{-s x} d \mathcal{G}(x, t)=e^{-\lambda t}+\int_{0}^{\infty} e^{-s x} g(x, t) d x=\exp \{\lambda t(\tilde{f}(s)-1)\} .
$$

Nie et al. (2015) consider finite time ruin probabilities for the risk model with capital injections. Their approach is based on the number of capital injections and the times between capital injections. They obtain a general expression for the distribution function of $T_{k}$, but are only able to implement their results if the joint density $w(u, y, t)$ (in the classical risk model) can be decomposed in a particular way. Our motivation is to find a more general expression, and we do this by considering the density function $w_{k}(u, t)$. A natural tool to find this is a Gerber-Shiu function. We therefore construct such a function, focussing on the time of ruin and the number of claims until ruin, denoted by $N_{T_{k}}$, which allows us to generalise some existing results on these two variables for the classical risk model. The reason for including $N_{T_{k}}$ in our analysis is that it allows us to see that the density $w_{k}(u, t)$ is expressed in terms of the joint density of $T_{k}$ and $N_{T_{k}}$ in a very transparent way.

\section{A Gerber-Shiu function}

As in Landriault et al. (2011), we define our Gerber-Shiu function in terms of $T_{k}, N_{T_{k}}$, and a general penalty function $\omega(x, y)$, defined for $x \geq k$ and $y>0$, as

$$
m_{r, \delta}(u)=E\left[r^{N_{T_{k}}} e^{-\delta T_{k}} \omega\left(U\left(T_{k}^{-}\right),\left|U\left(T_{k}\right)\right|\right) I\left(T_{k}<\infty\right) \mid U(0)=u\right],
$$

for $\delta \geq 0,0<r \leq 1$ and $u \geq k$, where $U\left(T_{k}^{-}\right)$is the surplus immediately prior to ruin. As in Landriault et al. (2011), we interpret $\delta$ as the parameter of a Laplace transform and $r$ as the parameter of a probability generating function. Further, $m_{r, \delta}(u)$ is defined to be 0 for $0 \leq u<k$.

Theorem 3.1 The Laplace transform of Gerber-Shiu function $m_{r, \delta}(u)$ satisfies

$$
\tilde{m}_{r, \delta}(s)=\frac{1}{c s-\lambda-\delta+r \lambda \tilde{f}(s)}\left(c e^{-s k} m_{r, \delta}(k)-r \lambda \int_{k}^{\infty} e^{-s u}(\bar{F}(u-k)-\bar{F}(u)) m_{r, \delta}(k) d u\right.
$$




$$
\left.-r \lambda \int_{k}^{\infty} e^{-s u} \int_{u}^{\infty} f(x) \omega(u, x-u) d x d u\right)
$$

Further,

$$
m_{r, \delta}(k)=\frac{\frac{r \lambda}{c} \int_{k}^{\infty} \int_{u}^{\infty} e^{-\rho(u-k)} f(x) \omega(u, x-u) d x d u}{1-\frac{r \lambda}{c} \int_{k}^{\infty} e^{-\rho(u-k)}(\bar{F}(u-k)-\bar{F}(u)) d u} .
$$

Proof 3.1 Using the standard argument of conditioning on the time and the amount of the first claim we obtain for $u \geq k$

$$
\begin{aligned}
m_{r, \delta}(u)= & \frac{r \lambda}{c} \int_{u}^{\infty} e^{-(\lambda+\delta)(\tau-u) / c}\left(\int_{0}^{\tau-k} f(x) m_{r, \delta}(\tau-x) d x+\int_{\tau-k}^{\tau} f(x) m_{r, \delta}(k) d x\right. \\
& \left.+\int_{\tau}^{\infty} f(x) \omega(\tau, x-\tau) d x\right) d \tau \\
= & \frac{r \lambda}{c} \int_{u}^{\infty} e^{-(\lambda+\delta)(\tau-u) / c} \gamma(\tau) d \tau
\end{aligned}
$$

where

$$
\gamma(u)=\int_{0}^{u-k} f(x) m_{r, \delta}(u-x) d x+\int_{u-k}^{u} f(x) m_{r, \delta}(k) d x+\zeta(u)
$$

and

$$
\zeta(u)=\int_{u}^{\infty} f(x) \omega(u, x-u) d x .
$$

Using the operator $T_{s}$ introduced by Dickson and Hipp (2001), and defined for an integrable function $f$ as

$$
T_{s} f(x)=\int_{x}^{\infty} e^{-s(u-x)} f(u) d u
$$

we have

$$
m_{r, \delta}(u)=\frac{r \lambda}{c} T_{\frac{\lambda+\delta}{c}} \gamma(u)
$$

Noting that $m_{r, \delta}(u)=0$ for $0 \leq u<k$ we have

$$
T_{s} m_{r, \delta}(k)=\int_{k}^{\infty} e^{-s(x-k)} m_{r, \delta}(x) d x=e^{s k} \tilde{m}_{r, \delta}(s),
$$

and similarly $T_{s} \gamma(k)=e^{s k} \tilde{\gamma}(s)$. Applying the Dickson-Hipp operator to equation (3.4) we obtain

$$
T_{s} m_{r, \delta}(k)=\frac{r \lambda}{c} T_{s} T_{\frac{\lambda+\delta}{c}} \gamma(k)=\frac{r \lambda}{c} \frac{T_{\frac{\lambda+\delta}{c}} \gamma(k)-T_{s} \gamma(k)}{s-\frac{\lambda+\delta}{c}},
$$

where we have used properties of the operator $T_{s}$ given in Dickson and Hipp (2001). Further,

$$
\begin{aligned}
T_{s} \gamma(k) & =\int_{k}^{\infty} e^{-s(u-k)} \gamma(u) d u \\
& =e^{s k} \int_{k}^{\infty} e^{-s u} \int_{0}^{u-k} f(x) m_{r, \delta}(u-x) d x d u+m_{r, \delta}(k) \int_{k}^{\infty} e^{-s(u-k)}(\bar{F}(u-k)-\bar{F}(u)) d u
\end{aligned}
$$




$$
\begin{aligned}
& +\int_{k}^{\infty} e^{-s(u-k)} \zeta(u) d u \\
= & e^{s k} \tilde{f}(s) \tilde{m}_{r, \delta}(s)+m_{r, \delta}(k) \int_{k}^{\infty} e^{-s(u-k)}(\bar{F}(u-k)-\bar{F}(u)) d u+T_{s} \zeta(k) \\
= & \tilde{f}(s) T_{s} m_{r, \delta}(k)+m_{r, \delta}(k) \int_{k}^{\infty} e^{-s(u-k)}(\bar{F}(u-k)-\bar{F}(u)) d u+T_{s} \zeta(k) .
\end{aligned}
$$

Substituting in (3.5) we obtain

$$
\begin{aligned}
T_{s} m_{r, \delta}(k)= & \frac{r \lambda}{c s-\lambda-\delta}\left(T_{\frac{\lambda+\delta}{c}} \gamma(k)-\tilde{f}(s) T_{s} m_{r, \delta}(k)\right. \\
& \left.-m_{r, \delta}(k) \int_{k}^{\infty} e^{-s(u-k)}(\bar{F}(u-k)-\bar{F}(u)) d u-T_{s} \zeta(k)\right)
\end{aligned}
$$

which can be written in terms of Laplace transforms as

$$
\begin{aligned}
e^{s k} \tilde{m}_{r, \delta}(s)= & \frac{r \lambda}{c s-\lambda-\delta}\left(\frac{c}{r \lambda} m_{r, \delta}(k)-\tilde{f}(s) e^{s k} \tilde{m}_{r, \delta}(s)\right. \\
& \left.-m_{r, \delta}(k) \int_{k}^{\infty} e^{-s(u-k)}(\bar{F}(u-k)-\bar{F}(u)) d u-\int_{k}^{\infty} e^{-s(u-k)} \zeta(u) d u\right) .
\end{aligned}
$$

Rearranging this identity we obtain formula (3.2).

To obtain formula (3.3) we first note from formula (41) of Landriault et al. (2011) that there exists $\rho \equiv \rho(\delta, r)$ which is the unique positive solution of $c s-\lambda-\delta+r \lambda \tilde{f}(s)=0$. Then, as $\rho$ is a zero of the denominator of the right-hand side of (3.2), it must also be a zero of the numerator, giving

$$
m_{r, \delta}(k)=\frac{\frac{r \lambda}{c} \int_{k}^{\infty} \int_{u}^{\infty} e^{-\rho(u-k)} f(x) \omega(u, x-u) d x d u}{1-\frac{r \lambda}{c} \int_{k}^{\infty} e^{-\rho(u-k)}(\bar{F}(u-k)-\bar{F}(u)) d u} .
$$

By choosing different forms of the penalty function, equations (3.2) and (3.3) can be inverted to obtain several ruin related quantities. In the next section we consider the ultimate ruin probability, and in Section 5 we consider the joint density of $T_{k}$ and $N_{T_{k}}$.

\section{The ultimate ruin probability}

We now consider the ultimate ruin probability $\psi_{k}(u)$, for $u \geq k$. Nie et al. (2011) obtain expressions for this probability in the cases $u=k$ and $u>k$ by using probabilistic arguments. We now show that their results can be obtained from formulae (3.2) and (3.3). Setting $r=1, \delta=0$ and $\omega(x, y)=1$ for $x \geq k$ and $y>0$ in expression (3.1), we see that $m_{r, \delta}(u)$ reduces to $\psi_{k}(u)$. Our first result is easily obtained.

Theorem 4.1 When the initial surplus is $k$ we have

$$
\psi_{k}(k)=\frac{\frac{\lambda}{c} \int_{k}^{\infty} \bar{F}(u) d u}{1-\frac{\lambda}{c} \int_{k}^{\infty}(\bar{F}(u-k)-\bar{F}(u)) d u}=\frac{\psi(0)-G(0, k)}{1-G(0, k)} .
$$

Proof 4.1 From Gerber and Shiu (1998) we know that $\rho=0$ when $r=1$ and $\delta=0$. The result immediately follows from formula (3.3). 
Theorem 4.2 When the initial surplus is $u>k$ we have

$$
\psi_{k}(u)=\psi(u-k)-G(u-k, k)\left[1-\psi_{k}(k)\right] .
$$

Proof 4.2 Using the fact that $\frac{c \phi(0)}{\tilde{\phi}(s)}=c s-\lambda+\lambda \tilde{f}(s)$ (e.g., Dickson (2005)) equation (3.2) becomes

$$
\begin{aligned}
\tilde{\psi}_{k}(s)= & \frac{\tilde{\phi}(s)}{c \phi(0)}\left(c e^{-s k} \psi_{k}(k)-\lambda \int_{k}^{\infty} e^{-s u}(\bar{F}(u-k)-\bar{F}(u)) \psi_{k}(k) d u\right. \\
& \left.-\lambda \int_{k}^{\infty} e^{-s u} \int_{u}^{\infty} f(x) d x d u\right) \\
= & e^{-s k} \frac{\tilde{\phi}(s)}{\phi(0)} \psi_{k}(k)-\frac{\lambda \tilde{\phi}(s)}{c \phi(0)} \psi_{k}(k) \int_{0}^{\infty} e^{-s(u+k)}(\bar{F}(u)-\bar{F}(u+k)) d u \\
& -\frac{\lambda \tilde{\phi}(s)}{c \phi(0)} \int_{0}^{\infty} e^{-s(u+k)} \bar{F}(u+k) d u \\
= & e^{-s k}\left(\frac{\tilde{\phi}(s)}{\phi(0)} \psi_{k}(k)-\frac{\tilde{\phi}(s)}{\phi(0)} \psi_{k}(k) \int_{0}^{\infty} e^{-s u}(g(0, u)-g(0, u+k)) d u\right. \\
& \left.-\frac{\tilde{\phi}(s)}{\phi(0)} \int_{0}^{\infty} e^{-s u} g(0, u+k) d u\right) .
\end{aligned}
$$

We now apply the following results from Dickson (1998):

$$
\tilde{g}(0, s)=\int_{0}^{\infty} e^{-s u} g(0, u) d u=1-\frac{\phi(0)}{s \tilde{\phi}(s)}
$$

and

$$
\tilde{G}(s, k)=\int_{0}^{\infty} e^{-s u} G(u, k) d u=\frac{\tilde{\Phi}(s, k)}{1-\tilde{g}(0, s)}=\frac{\tilde{\Phi}(s, k) s \tilde{\phi}(s)}{\phi(0)}
$$

where $\tilde{\Phi}(s, k)=\int_{0}^{\infty} e^{-s u} \Phi(u, k) d u$, and $\Phi(u, k)=\int_{u}^{u+k} g(0, x) d x$. Next, we can use properties of the Laplace transform of a derivative to write

$$
\tilde{G}(s, k)=\frac{\tilde{\phi}(s)}{\phi(0)}\left(\tilde{\Phi}^{\prime}(s, k)+G(0, k)\right)
$$

with $\tilde{\Phi}^{\prime}(s, k)=\int_{0}^{\infty} e^{-s u} \Phi^{\prime}(u, k) d u=s \tilde{\Phi}(s, k)-G(0, k)$. Further,

$$
\int_{0}^{\infty} e^{-s u} \Phi^{\prime}(u, k) d u=\int_{0}^{\infty} e^{-s u}(g(0, u+k)-g(0, u)) d u .
$$

Using these results we can write formula (4.3) as

$$
\begin{aligned}
\tilde{\psi}_{k}(s)=e^{-s k}\left(\frac{\tilde{\phi}(s)}{\phi(0)} \psi_{k}(k)+\frac{\tilde{\phi}(s)}{\phi(0)} \psi_{k}(k) \tilde{\Phi}^{\prime}(s, k)\right. \\
\left.\quad-\frac{\tilde{\phi}(s)}{\phi(0)} \int_{0}^{\infty} e^{-s u}(g(0, u+k)-g(0, u)+g(0, u)) d u\right)
\end{aligned}
$$




$$
=e^{-s k}\left(\frac{\tilde{\phi}(s)}{\phi(0)} \psi_{k}(k)+\frac{\tilde{\phi}(s)}{\phi(0)} \psi_{k}(k) \tilde{\Phi}^{\prime}(s, k)-\frac{\tilde{\phi}(s)}{\phi(0)} \tilde{\Phi}^{\prime}(s, k)-\frac{\tilde{\phi}(s)}{\phi(0)} \tilde{g}(0, s)\right) .
$$

By rearranging formula (4.4) and substituting for $\tilde{\Phi}^{\prime}(s, k)$ in (4.5) we get

$$
\begin{aligned}
\tilde{\psi}_{k}(s)=e^{-s k}\left(\frac{\tilde{\phi}(s)}{\phi(0)} \psi_{k}(k)+\psi_{k}(k)\left[\tilde{G}(s, k)-\frac{\tilde{\phi}(s)}{\phi(0)} G(0, k)\right]\right. \\
\left.-\left[\tilde{G}(s, k)-\frac{\tilde{\phi}(s)}{\phi(0)} G(0, k)\right]-\frac{\tilde{\phi}(s)}{\phi(0)}\left[1-\frac{\phi(0)}{s \tilde{\phi}(s)}\right]\right) \\
=e^{-s k}\left(\frac{\tilde{\phi}(s)}{\phi(0)}\left[\psi_{k}(k)-\psi_{k}(k) G(0, k)+G(0, k)-1+\frac{\phi(0)}{s \tilde{\phi}(s)}\right]\right. \\
\left.-\tilde{G}(s, k)\left[1-\psi_{k}(k)\right]\right) .
\end{aligned}
$$

Inserting the right-hand side of equation (4.1) for $\psi_{k}(k)$ in the first square bracket gives

$$
\begin{aligned}
\tilde{\psi}_{k}(s) & =e^{-s k}\left(\frac{\tilde{\phi}(s)}{\phi(0)}\left[-\phi(0)+\frac{\phi(0)}{s \tilde{\phi}(s)}\right]-\tilde{G}(s, k)\left[1-\psi_{k}(k)\right]\right) \\
& =e^{-s k}[1 / s-\tilde{\phi}(s)]-e^{-s k} \tilde{G}(s, k)\left[1-\psi_{k}(k)\right] .
\end{aligned}
$$

Inversion of $\tilde{\psi}_{k}(s)$ yields

$$
\psi_{k}(u)=\psi(u-k)-G(u-k, k)\left[1-\psi_{k}(k)\right] .
$$

Formulae (4.1) and (4.6) appear in Nie et al. (2011) where the derivation is simpler. So, although Gerber-Shiu functions are very useful, they are not always the most efficient tools. However, we show in the next section that our Gerber-Shiu function can be used to obtain more general results relating to finite time ruin than those given in Nie et al. (2015).

\section{The joint density of $T_{k}$ and $N_{T_{k}}$}

We now consider the joint density of the time of ruin and the number of claims until ruin by setting $0<r<1, \delta>0$ and $\omega(x, y)=1$ for $x \geq k$ and $y>0$ in expression (3.1). (For convenience, we use the term joint density throughout when referring to two variables, even if one of the variables is discrete.) In this case the Gerber-Shiu function is

$$
m_{r, \delta}(u)=E\left[r^{N_{T_{k}}} e^{-\delta T_{k}} I\left(T_{k}<\infty\right) \mid U(0)=u\right]=\sum_{n=1}^{\infty} r^{n} \int_{0}^{\infty} e^{-\delta t} w_{k}(u, n, t) d t
$$

where $w_{k}(u, n, t)$ denotes the joint density of $T_{k}$ and $N_{T_{k}}$, given initial surplus $u$, defined for $n=1,2,3, \ldots$ and $t>0$. 


\subsection{General results}

Theorem 5.1 When the initial surplus is $k$, the joint density of $T_{k}$ and $N_{T_{k}}$ is given by

$$
w_{k}(k, 1, t)=\lambda e^{-\lambda t} \bar{F}_{0,1}(c t)=\lambda e^{-\lambda t} \bar{F}(c t+k),
$$

and for $n=1,2,3, \ldots$

$$
\begin{aligned}
w_{k}(k, n+1, t)= & \frac{\lambda^{n+1}}{c^{n}} \sum_{m=0}^{n}(-1)^{m}\left(\begin{array}{c}
n \\
m
\end{array}\right) e^{-\lambda t} \bar{F}_{m, n+1}(c t) \\
& +\sum_{j=0}^{n-1} e^{-\lambda t} \frac{\lambda^{n+1}}{c^{j+1}} \frac{t^{n-j-1}}{(n-j) !} \sum_{m=0}^{j}(-1)^{m}\left(\begin{array}{c}
j \\
m
\end{array}\right) \int_{0}^{c t} y f^{(n-j) *}(c t-y) \bar{F}_{m, j+1}(y) d y,
\end{aligned}
$$

where for $n=1,2,3, \ldots$ and $m=0,1,2, \ldots, n-2$,

$$
\bar{F}_{m, n}(u)=\int_{0}^{u} A^{(n-1-m) *}(x) B_{k}^{(m+1) *}(u-x) d x,
$$

$A(x)=\bar{F}(x)$ and $B_{k}(x)=\bar{F}(x+k)$, and $\bar{F}_{n-1, n}(u)=B_{k}^{n *}(u)$.

Proof 5.1 We start by re-writing formula (3.3) as

$$
m_{r, \delta}(k)=\frac{r \lambda}{c} \int_{k}^{\infty} e^{-\rho(u-k)} \bar{F}(u) d u \sum_{n=0}^{\infty}\left(\frac{r \lambda}{c} \int_{k}^{\infty} e^{-\rho(u-k)}(\bar{F}(u-k)-\bar{F}(u)) d u\right)^{n},
$$

and using the binomial expansion we obtain

$m_{r, \delta}(k)=\sum_{n=1}^{\infty} \frac{(r \lambda)^{n}}{c^{n}} \sum_{m=0}^{n-1}(-1)^{m}\left(\begin{array}{c}n-1 \\ m\end{array}\right)\left(\int_{0}^{\infty} e^{-\rho u} \bar{F}(u) d u\right)^{n-1-m}\left(\int_{0}^{\infty} e^{-\rho u} \bar{F}(u+k) d u\right)^{m+1}$,

giving

$$
m_{r, \delta}(k)=\sum_{n=1}^{\infty} \frac{(r \lambda)^{n}}{c^{n}} \sum_{m=0}^{n-1}(-1)^{m}\left(\begin{array}{c}
n-1 \\
m
\end{array}\right) \int_{0}^{\infty} e^{-\rho u} \bar{F}_{m, n}(u) d u .
$$

To invert formula (5.4) we use formula (44) of Landriault et al. (2011) to obtain

$$
\begin{aligned}
\sum_{n=1}^{\infty} r^{n} w_{k}(k, n, t)= & \sum_{n=1}^{\infty} \frac{(r \lambda)^{n}}{c^{n}} \sum_{m=0}^{n-1}(-1)^{m}\left(\begin{array}{c}
n-1 \\
m
\end{array}\right)\left(e^{-\lambda t} c \bar{F}_{m, n}(c t)\right. \\
& \left.+\sum_{j=1}^{\infty} e^{-\lambda t} \frac{(r \lambda)^{j}}{j !} t^{j-1} \int_{0}^{c t} y f^{j *}(c t-y) \bar{F}_{m, n}(y) d y\right) \\
= & \sum_{n=1}^{\infty} \frac{(r \lambda)^{n}}{c^{n}} \sum_{m=0}^{n-1}(-1)^{m}\left(\begin{array}{c}
n-1 \\
m
\end{array}\right) e^{-\lambda t} c \bar{F}_{m, n}(c t) \\
& +\sum_{n=1}^{\infty} r^{n+1} \sum_{j=0}^{n-1} e^{-\lambda t} \frac{\lambda^{n+1}}{c^{j+1}} \frac{t^{n-j-1}}{(n-j) !} \sum_{m=0}^{j}(-1)^{m}\left(\begin{array}{c}
j \\
m
\end{array}\right)
\end{aligned}
$$




$$
\times \int_{0}^{c t} y f^{(n-j) *}(c t-y) \bar{F}_{m, j+1}(y) d y
$$

Formulae (5.1) and (5.2) then follow by equating coefficients of powers of $r$ in equation (5.5).

We remark that if we set $r=1$ in (5.5) then we obtain an expression for the density of the time of ruin, $w_{k}(k, t)$.

Theorem 5.2 When $u>k$,

$$
w_{k}(u, 1, t)=\lambda e^{-\lambda t} \bar{F}(u+c t),
$$

and for $n=1,2,3, \ldots$ we have

$$
\begin{aligned}
w_{k}(u, n+1, t)= & e^{-\lambda t} \frac{(\lambda t)^{n}}{n !} \int_{0}^{u+c t-k} f^{n *}(u+c t-x-k) \lambda \bar{F}(x+k) d x \\
& +\int_{0}^{t} w_{k}(k, n, t-\tau) \lambda e^{-\lambda \tau}(\bar{F}(u+c \tau-k)-\bar{F}(u+c \tau)) d \tau \\
& +\sum_{m=1}^{n-1} \int_{0}^{t} e^{-\lambda \tau} \frac{(\lambda \tau)^{m}}{m !} \int_{0}^{u+c \tau-k} f^{m *}(u+c \tau-k-x) \\
& \times \lambda(\bar{F}(x)-\bar{F}(x+k)) d x w_{k}(k, n-m, t-\tau) d \tau \\
& -c \sum_{m=1}^{n} \int_{0}^{t} e^{-\lambda \tau} \frac{(\lambda \tau)^{m}}{m !} f^{m *}(u+c \tau-k) w_{k}(k, n+1-m, t-\tau) d \tau,
\end{aligned}
$$

with the usual convention that $\sum_{j=a}^{b}=0$ if $b<a$.

Proof 5.2 We start by noting that

$$
\tilde{m}_{r, \delta}(s)=\int_{k}^{\infty} e^{-s u} m_{r, \delta}(u) d u=\sum_{n=1}^{\infty} r^{n} \int_{k}^{\infty} e^{-s u} \int_{0}^{\infty} e^{-\delta t} w_{k}(u, n, t) d t d u .
$$

Next, we re-write formula (3.2) as

$$
\begin{aligned}
\tilde{m}_{r, \delta}(s)= & \frac{1}{\delta+\lambda-r \lambda \tilde{f}(s)-c s}\left(-c e^{-s k} m_{r, \delta}(k)\right. \\
& \left.+r \lambda m_{r, \delta}(k) \int_{k}^{\infty} e^{-s u}(\bar{F}(u-k)-\bar{F}(u)) d u+r \lambda \int_{k}^{\infty} e^{-s u} \bar{F}(u) d u\right),
\end{aligned}
$$

and our approach is to invert first with respect to $\delta$, and then with respect to s. Our derivation uses ideas in Panjer and Willmot (1992) and is based on the Laplace transform of $S(t)$; see also Willmot (2015). Our starting point is to define a function $A_{r}(u, t)$ whose Laplace transform with respect to $u$ is $\tilde{A}_{r}(s, t)$, and its bivariate Laplace transform is given by

$$
\tilde{\tilde{A}}_{r}(s, \delta)=\int_{0}^{\infty} e^{-\delta t} \tilde{A}_{r}(s, t) d t=\frac{1}{\delta+\lambda-r \lambda \tilde{f}(s)-c s} .
$$


Inverting this expression with respect to $\delta$ gives $\tilde{A}_{r}(s, t)=\exp \{\lambda r t(\tilde{f}(s)-1)+\lambda r t-\lambda t+$ $s c t\}$. From the Laplace transform of $S(t)$ in formula (2.1) we deduce that $\tilde{A}_{r}(s, t)$ is the product of the Laplace transform of $S_{r}(t)$, where $S_{r}(t)$ is as $S(t)$ except that its Poisson parameter is $\lambda r t$ (and its density is denoted by $g_{r}$ ), and the term $\exp \{\lambda t(r-1)+s c t\}$. Hence we can write formula (5.8) as

$$
\tilde{m}_{r, \delta}(s)=\tilde{\tilde{A}}_{r}(s, \delta)\left(-c e^{-s k} m_{r, \delta}(k)+r \lambda m_{r, \delta}(k) \tilde{C}(s)+r \lambda \tilde{B}(s)\right),
$$

where $C(u)=(\bar{F}(u-k)-\bar{F}(u)) I\{u>k\}$ and $B(u)=\bar{F}(u) I\{u>k\}$. Inverting equation (5.9) with respect to $\delta$ yields

$$
\begin{aligned}
& \sum_{n=1}^{\infty} r^{n} \int_{k}^{\infty} e^{-s u} w_{k}(u, n, t) d u \\
= & -c \sum_{n=1}^{\infty} r^{n} e^{-s k} \int_{0}^{t} w_{k}(k, n, \tau) \exp \{\lambda r(t-\tau)(\tilde{f}(s)-1)+\lambda(t-\tau)(r-1)+s c(t-\tau)\} d \tau \\
& +\lambda r \tilde{C}(s) \sum_{n=1}^{\infty} r^{n} \int_{0}^{t} w_{k}(k, n, \tau) \exp \{\lambda r(t-\tau)(\tilde{f}(s)-1)+\lambda(t-\tau)(r-1)+s c(t-\tau)\} d \tau \\
& +\lambda r \tilde{B}(s) \exp \{\lambda r t(\tilde{f}(s)-1)+\lambda t(r-1)+s c t\} .
\end{aligned}
$$

Multiplying both sides by $e^{-s c t}$ gives

$$
\begin{aligned}
& \sum_{n=1}^{\infty} r^{n} e^{-s c t} \int_{k}^{\infty} e^{-s u} w_{k}(u, n, t) d u \\
= & -c \sum_{n=1}^{\infty} r^{n} e^{-s k} \int_{0}^{t} w_{k}(k, n, \tau) \exp \{\lambda r(t-\tau)(\tilde{f}(s)-1)+\lambda(t-\tau)(r-1)-s c \tau\} d \tau \\
& +\lambda r \tilde{C}(s) \sum_{n=1}^{\infty} r^{n} \int_{0}^{t} w_{k}(k, n, \tau) \exp \{\lambda r(t-\tau)(\tilde{f}(s)-1)+\lambda(t-\tau)(r-1)-s c \tau\} d \tau \\
& +\lambda r \tilde{B}(s) \exp \{\lambda r t(\tilde{f}(s)-1)+\lambda t(r-1)\} .
\end{aligned}
$$

The left-hand side of equation (5.10) can be written as

$$
\sum_{n=1}^{\infty} r^{n} \int_{k}^{\infty} e^{-s(u+c t)} w_{k}(u, n, t) d u=\sum_{n=1}^{\infty} r^{n} \int_{c t+k}^{\infty} e^{-s u} w_{k}(u-c t, n, t) d u,
$$

and (minus) the first term on the right-hand side as

$$
\begin{aligned}
& c \sum_{n=1}^{\infty} r^{n} e^{-s k} \int_{0}^{t} w_{k}(k, n, \tau) \exp \{\lambda r(t-\tau)(\tilde{f}(s)-1)+\lambda(t-\tau)(r-1)-s c \tau\} d \tau \\
= & c \sum_{n=1}^{\infty} r^{n} e^{-s k} \int_{0}^{t} w_{k}(k, n, \tau)\left(\tilde{g}_{r}(s, t-\tau)+e^{-\lambda r(t-\tau)}\right) e^{\lambda(t-\tau)(r-1)-s c \tau} d \tau \\
= & c \sum_{n=1}^{\infty} r^{n} \int_{0}^{t} w_{k}(k, n, \tau) \int_{0}^{\infty} e^{-s(x+c \tau+k)} g_{r}(x, t-\tau) e^{\lambda(t-\tau)(r-1)} d x d \tau \\
& +c \sum_{n=1}^{\infty} r^{n} \int_{0}^{t} w_{k}(k, n, \tau) e^{-s(c \tau+k)} e^{-\lambda(t-\tau)} d \tau
\end{aligned}
$$




$$
\begin{aligned}
= & c \sum_{n=1}^{\infty} r^{n} \int_{0}^{t} w_{k}(k, n, \tau) \int_{0}^{\infty} e^{-s(x+c \tau+k)} \sum_{m=1}^{\infty} e^{-\lambda(t-\tau)} \frac{(\lambda r(t-\tau))^{m}}{m !} f^{m *}(x) d x d \tau \\
& +\sum_{n=1}^{\infty} r^{n} \int_{k}^{c t+k} w_{k}(k, n,(u-k) / c) e^{-s u} e^{-\lambda(t-(u-k) / c)} d u \\
= & c \sum_{n=1}^{\infty} r^{n} \int_{0}^{t} w_{k}(k, n, \tau) \int_{c \tau+k}^{\infty} e^{-s u} \sum_{m=1}^{\infty} e^{-\lambda(t-\tau)} \frac{(\lambda r(t-\tau))^{m}}{m !} f^{m *}(u-c \tau-k) d u d \tau \\
& +\sum_{n=1}^{\infty} r^{n} \int_{k}^{c t+k} w_{k}(k, n,(u-k) / c) e^{-s u} e^{-\lambda(t-(u-k) / c)} d u \\
= & c \sum_{n=1}^{\infty} r^{n} \int_{k}^{c t+k} e^{-s u} \int_{0}^{(u-k) / c} w_{k}(k, n, \tau) \sum_{m=1}^{\infty} e^{-\lambda(t-\tau)} \frac{(\lambda r(t-\tau))^{m}}{m !} f^{m *}(u-c \tau-k) d \tau d u \\
& +c \sum_{n=1}^{\infty} r^{n} \int_{c t+k}^{\infty} e^{-s u} \int_{0}^{t} w_{k}(k, n, \tau) \sum_{m=1}^{\infty} e^{-\lambda(t-\tau)} \frac{(\lambda r(t-\tau))^{m}}{m !} f^{m *}(u-c \tau-k) d \tau d u \\
& +\sum_{n=1}^{\infty} r^{n} \int_{k}^{c t+k} e^{-s u} w_{k}(k, n,(u-k) / c) e^{-\lambda(t-(u-k) / c)} d u .
\end{aligned}
$$

We can treat the second term of the right-hand side of equation (5.10) similarly to show that

$$
\begin{aligned}
& \lambda r \tilde{C}(s) \sum_{n=1}^{\infty} r^{n} \int_{0}^{t} w_{k}(k, n, \tau) \exp \{\lambda r(t-\tau)(\tilde{f}(s)-1)+\lambda(t-\tau)(r-1)-s c \tau\} d \tau \\
= & \lambda r \sum_{n=1}^{\infty} r^{n} \int_{c t}^{\infty} e^{-s u} \int_{0}^{t} w_{k}(k, n, \tau) \int_{0}^{u-c \tau-k} \sum_{m=1}^{\infty} e^{-\lambda(t-\tau)} \frac{(\lambda r(t-\tau))^{m}}{m !} f^{m *}(x) \\
& \times(\bar{F}(u-c \tau-k-x)-\bar{F}(u-c \tau-x)) d x d \tau d u \\
& +\lambda r \sum_{n=1}^{\infty} r^{n} \int_{0}^{c t} e^{-s u} \int_{0}^{u / c} w_{k}(k, n, \tau) \int_{0}^{u-c \tau} \sum_{m=1}^{\infty} e^{-\lambda(t-\tau)} \frac{(\lambda r(t-\tau))^{m}}{m !} f^{m *}(x) C(u-c \tau-x) d x d \tau d u \\
& +\lambda r \sum_{n=1}^{\infty} r^{n} \int_{c t}^{\infty} e^{-s u} \int_{0}^{t} w_{k}(k, n, \tau) \\
& \times(\bar{F}(u-c \tau-k)-\bar{F}(u-c \tau)) I(u-c \tau>k) e^{-\lambda(t-\tau)} d \tau d u \\
& +\lambda r \sum_{n=1}^{\infty} r^{n} \int_{0}^{c t} e^{-s u} \int_{0}^{u / c} w_{k}(k, n, \tau) C(u-c \tau) e^{-\lambda(t-\tau)} d \tau d u .
\end{aligned}
$$

Finally the third term on the right-hand side of equation (5.10) can be expressed as

$$
\int_{0}^{\infty} e^{-s u}\left(\lambda r e^{-\lambda t} \bar{F}(u) I(u>k)+\lambda r \sum_{n=1}^{\infty} e^{-\lambda t} \frac{(\lambda r t)^{n}}{n !} \int_{0}^{u-k} \bar{F}(x+k) f^{n *}(u-x-k) d x\right) d u .
$$

By equating the coefficients of $e^{-s u}$ for $u>c t+k$ we obtain

$$
\begin{aligned}
\sum_{n=1}^{\infty} r^{n} w_{k}(u-c t, n, t)= & \lambda r e^{-\lambda t} \bar{F}(u) \\
& +\lambda r \sum_{n=1}^{\infty} e^{-\lambda t} \frac{(\lambda r t)^{n}}{n !} \int_{0}^{u-k} \bar{F}(x+k) f^{n *}(u-x-k) d x
\end{aligned}
$$




$$
\begin{aligned}
& +\sum_{n=1}^{\infty} r^{n+1} \int_{0}^{t} w_{k}(k, n, \tau)\left[\lambda e^{-\lambda(t-\tau)}(\bar{F}(u-k-c \tau)-\bar{F}(u-c \tau))\right. \\
& +\lambda \sum_{m=1}^{\infty} e^{-\lambda(t-\tau)} \frac{[\lambda r(t-\tau)]^{m}}{m !} \int_{0}^{u-c \tau-k} f^{m *}(x) \\
& \times(\bar{F}(u-k-c \tau-x)-\bar{F}(u-c \tau-x)) d x] d \tau \\
& -c \sum_{n=1}^{\infty} r^{n} \sum_{m=1}^{\infty} \int_{0}^{t} w_{k}(k, n, \tau) e^{-\lambda(t-\tau)} \frac{[\lambda r(t-\tau)]^{m}}{m !} f^{m *}(u-c \tau-k) d \tau .
\end{aligned}
$$

This results in

$$
\begin{aligned}
\sum_{n=1}^{\infty} r^{n} w_{k}(u, n, t)= & \lambda r e^{-\lambda t} \bar{F}(u+c t) \\
& +\lambda r \sum_{n=1}^{\infty} e^{-\lambda t} \frac{(\lambda r t)^{n}}{n !} \int_{0}^{u+c t-k} \bar{F}(x+k) f^{n *}(u+c t-x-k) d x \\
& +\sum_{n=1}^{\infty} r^{n+1} \int_{0}^{t} w_{k}(k, n, t-\tau)\left[\lambda e^{-\lambda \tau}(\bar{F}(u+c \tau-k)-\bar{F}(u+c \tau))\right. \\
& +\lambda \sum_{m=1}^{\infty} e^{-\lambda \tau} \frac{(r \lambda \tau)^{m}}{m !} \int_{0}^{u+c \tau-k} f^{m *}(x) \\
& \times(\bar{F}(u+c \tau-k-x)-\bar{F}(u+c \tau-x)) d x] d \tau \\
& -c \sum_{n=1}^{\infty} r^{n} \sum_{m=1}^{\infty} \int_{0}^{t} w_{k}(k, n, t-\tau) e^{-\lambda \tau} \frac{(r \lambda \tau)^{m}}{m !} f^{m *}(u+c \tau-k) d \tau
\end{aligned}
$$

To obtain $w_{k}(u, n, t)$ we proceed as follows. We rewrite expression (5.11) as

$$
\begin{aligned}
\sum_{n=1}^{\infty} r^{n} w_{k}(u, n, t)= & \lambda r e^{-\lambda t} \bar{F}(u+c t) \\
& +\sum_{n=1}^{\infty} r^{n+1} e^{-\lambda t} \frac{(\lambda t)^{n}}{n !} \int_{0}^{u+c t-k} f^{n *}(u+c t-x-k) \lambda \bar{F}(x+k) d x \\
& +\sum_{n=1}^{\infty} r^{n+1} \int_{0}^{t} w_{k}(k, n, t-\tau) \lambda e^{-\lambda \tau}(\bar{F}(u+c \tau-k)-\bar{F}(u+c \tau)) d \tau \\
& +\sum_{n=1}^{\infty} r^{n+1} \int_{0}^{t} w_{k}(k, n, t-\tau) \lambda \sum_{m=1}^{\infty} r^{m} e^{-\lambda \tau} \frac{(\lambda \tau)^{m}}{m !} \\
& \times \int_{0}^{u+c \tau-k} f^{m *}(x)(\bar{F}(u+c \tau-k-x)-\bar{F}(u+c \tau-x)) d x d \tau \\
& -c \sum_{n=1}^{\infty} r^{n} \sum_{m=1}^{\infty} r^{m} \int_{0}^{t} e^{-\lambda \tau} \frac{(\lambda \tau)^{m}}{m !} f^{m *}(u+c \tau-k) w_{k}(k, n, t-\tau) d \tau
\end{aligned}
$$

Applying the Cauchy product $\sum_{n=1}^{\infty} a_{n} \sum_{m=1}^{\infty} b_{m}=\sum_{n=1}^{\infty} \sum_{m=1}^{n} a_{n+1-m} b_{m}$ to the double 
summations in (5.12) we have

$$
\begin{aligned}
\sum_{n=1}^{\infty} r^{n} w_{k}(u, n, t)= & \lambda r e^{-\lambda t} \bar{F}(u+c t) \\
& +\sum_{n=1}^{\infty} r^{n+1} e^{-\lambda t} \frac{(\lambda t)^{n}}{n !} \int_{0}^{u+c t-k} f^{n *}(u+c t-x-k) \lambda \bar{F}(x+k) d x \\
& +\sum_{n=1}^{\infty} r^{n+1} \int_{0}^{t} w_{k}(k, n, t-\tau) \lambda e^{-\lambda \tau}(\bar{F}(u+c \tau-k)-\bar{F}(u+c \tau)) d \tau \\
& +\sum_{n=1}^{\infty} r^{n+2} \sum_{m=1}^{n} \int_{0}^{t} w_{k}(k, n+1-m, t-\tau) e^{-\lambda \tau} \frac{(\lambda \tau)^{m}}{m !} \\
& \times \int_{0}^{u+c \tau-k} f^{m *}(x) \lambda(\bar{F}(u+c \tau-k-x)-\bar{F}(u+c \tau-x)) d x d \tau \\
& -c \sum_{n=1}^{\infty} r^{n+1} \sum_{m=1}^{n} \int_{0}^{t} e^{-\lambda \tau} \frac{(\lambda \tau)^{m}}{m !} f^{m *}(u+c \tau-k) w_{k}(k, n+1-m, t-\tau) d \tau .
\end{aligned}
$$

Formulae (5.6) and (5.7) then follow by equating coefficients of powers of $r$ in equation (5.13).

Formula (5.7) generalises formula (8) of Dickson (2012) for the joint density of the time of ruin and the number of claims until ruin in the classical risk model - if we set $k=0$ we recover the result for the classical risk model.

Further, as $w_{k}(u, t)=\sum_{n=1}^{\infty} w_{k}(u, n, t)$, we obtain the following result.

Theorem 5.3 For $u>k$, the density of $T_{k}$ is

$$
\begin{aligned}
w_{k}(u, t)= & \lambda e^{-\lambda t} \bar{F}(u+c t)+\lambda \int_{0}^{u+c t-k} \bar{F}(x+k) g(u+c t-x-k, t) d x \\
& +\int_{0}^{t} w_{k}(k, t-\tau)\left[\lambda e^{-\lambda \tau}(\bar{F}(u+c \tau-k)-\bar{F}(u+c \tau))\right. \\
& \left.+\int_{0}^{u+c \tau-k} g(u+c \tau-k-x, \tau) \lambda(\bar{F}(x)-\bar{F}(x+k)) d x\right] d \tau \\
& -c \int_{0}^{t} w_{k}(k, t-\tau) g(u+c \tau-k, \tau) d \tau .
\end{aligned}
$$

Formula (5.14) generalises formula (5) of Dickson (2007) for the density of the time of ruin in the classical risk model. Once again, setting $k=0$ gives the result for the classical risk model.

To understand formula (5.14), it is helpful to consider realisations of the surplus process that are representative of the different terms in the formula. Interpretation of components of formula (5.14) is similar to that given in Dickson (2007). However, there is one important difference. Consider

$$
g(u+c \tau-k-x, \tau) \lambda(\bar{F}(x)-\bar{F}(x+k)) w_{k}(k, t-\tau) .
$$

This term is associated with (i) an aggregate claim amount of $u+c \tau-k-x$ at time $\tau$, resulting in a surplus of $x+k$ at time $\tau$ in a classical risk model, (ii) a claim whose 
amount is between $x$ and $x+k$ at time $\tau$ (so that a capital injection occurs), and (iii) ruin occurring after a further time period of $t-\tau$ with the possibility of capital injections in this time period. Note that the term $g(u+c \tau-k-x, \tau)$ refers to aggregate claims in a classical risk model, not the risk model with capital injections, and this term includes the possibility that a realisation of a classical risk model which is at level $x+k$ at time $\tau$ has fallen below $k$ prior to time $\tau$. Figures 5.1 and 5.2 show realisations of the surplus process representing the first two terms in formula (5.14) where the surplus immediately prior to ruin is above $k$. Figures 5.3 and 5.4 show realisations of the surplus process representing the second integral term, with a capital injection occurring at time $\tau$. In these realisations, the surplus prior to time $\tau$ is always above $k$, and we distinguish between there being further capital injections after time $\tau$ (Figure 5.3) and no further capital injections (Figure 5.4). Figures 5.5 and 5.6 are as Figures 5.3 and 5.4 except that the surplus falls below $k$ prior to time $\tau$. Figures 5.7 and 5.8 show realisations of the surplus process representing the final term in formula (5.14). In these, $\tau$ is the last time at which the (classical) surplus process upcrosses through $k$, and we have distinguished between the cases where there are capital injections after time $\tau$ (Figure 5.7) and there are no capital injections after time $\tau$ (Figure 5.8). Only Figures 5.1, 5.3 and 5.4 show realisations of the surplus process with capital injections that result in ruin at time $t$. As in Prabhu's (1961) formula, the final term in formula (5.14) is a compensation term. Realisations like those in Figure 5.2 are compensated for by realisations such as that in Figure 5.8. Similarly, realisations such as those in Figures 5.5 and 5.6 are compensated for by realisations such as that in Figure 5.7. To see this, note that there is a last time (before time $\tau$ ) at which the surplus process upcrosses through $k$ in Figures 5.5 and 5.6.

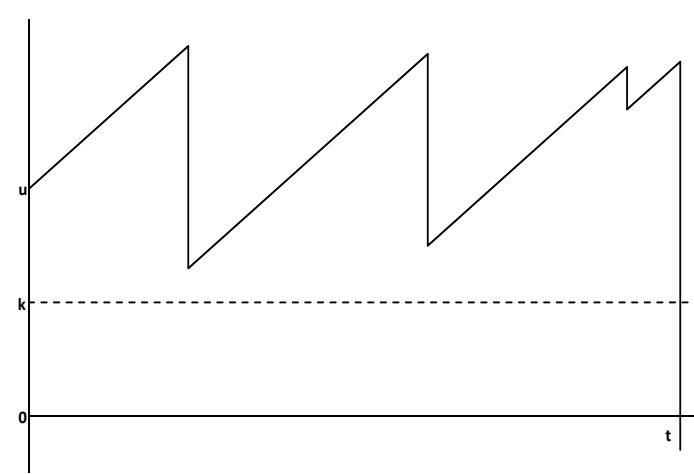

Figure 5.1: Classical surplus process always above $k$ prior to $t$

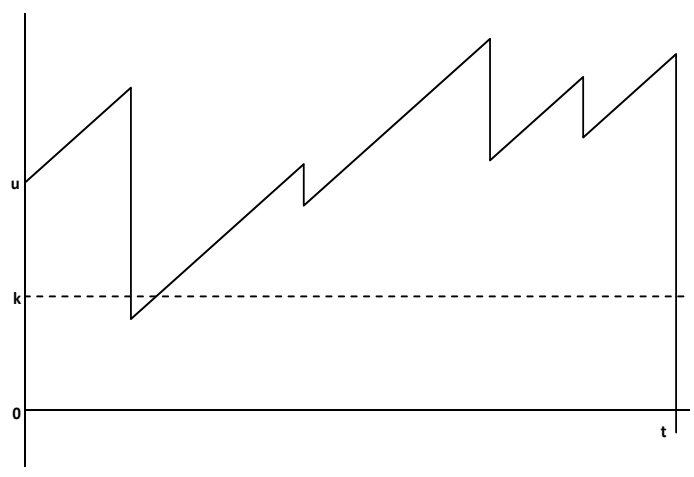

Figure 5.2: Classical surplus process below $k$ prior to $t$ 


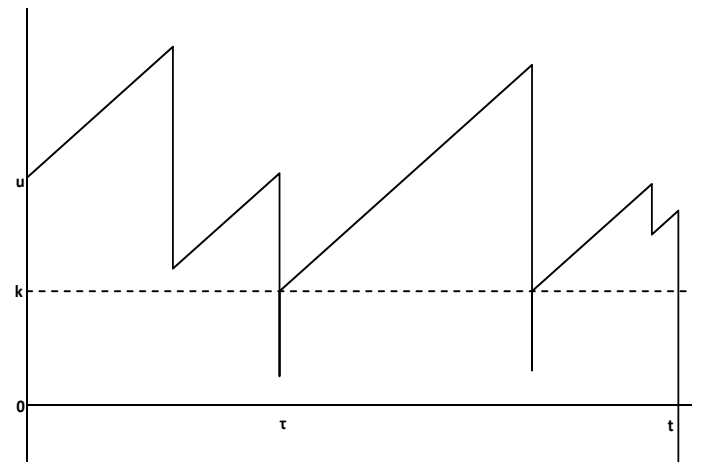

Figure 5.3: Classical surplus process above $k$ prior to capital injection at $\tau$; capital injected after $\tau$

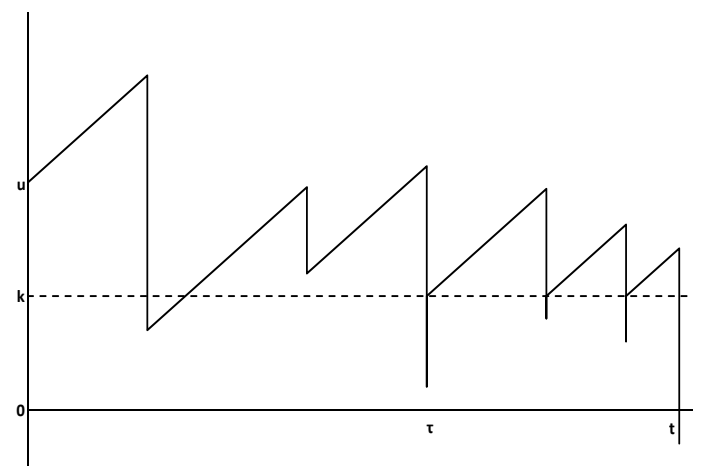

Figure 5.5: Classical surplus process below $k$ prior to capital injection at $\tau$; capital injected after $\tau$

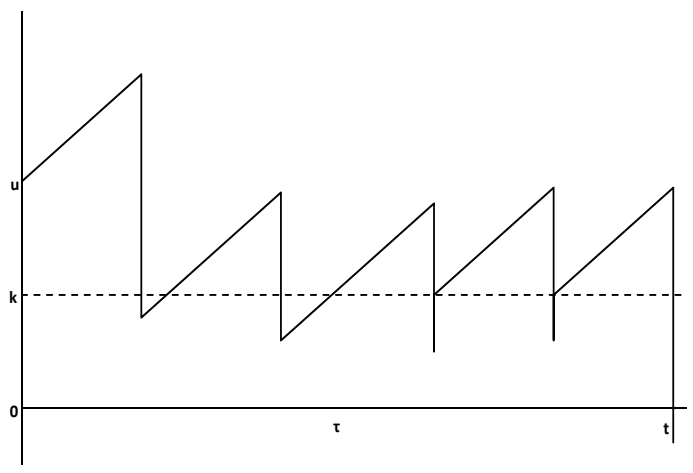

Figure 5.7: Classical surplus process uncrosses $k$ at $\tau$; ruin at $t$ with capital injections between $\tau$ and $t$.

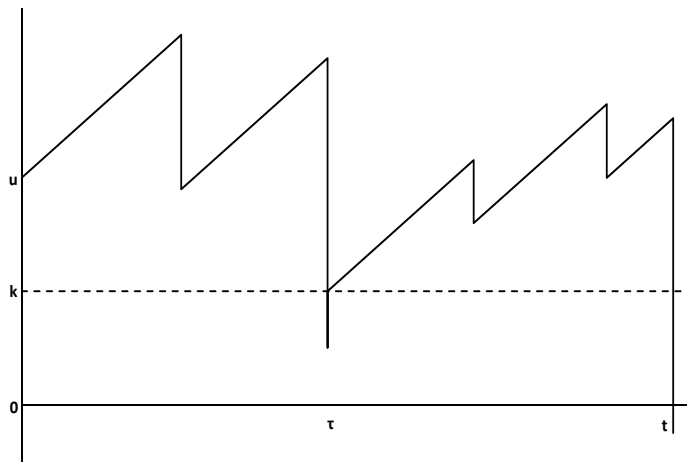

Figure 5.4: Classical surplus process above $k$ prior to capital injection at $\tau$; no capital injected after $\tau$

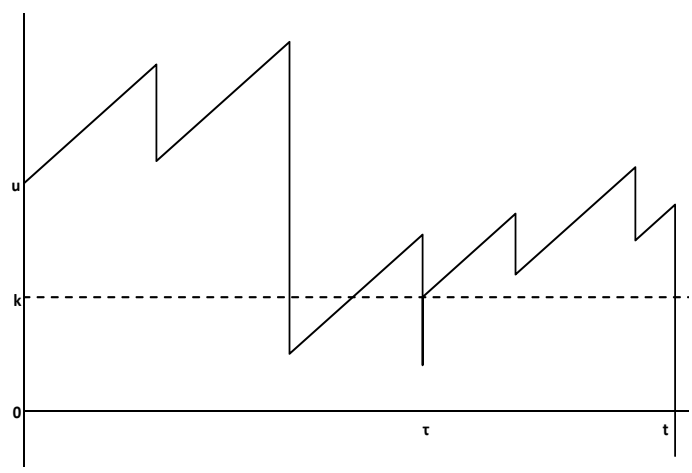

Figure 5.6: Classical surplus process below $k$ prior to capital injection at $\tau$; no capital injected after $\tau$

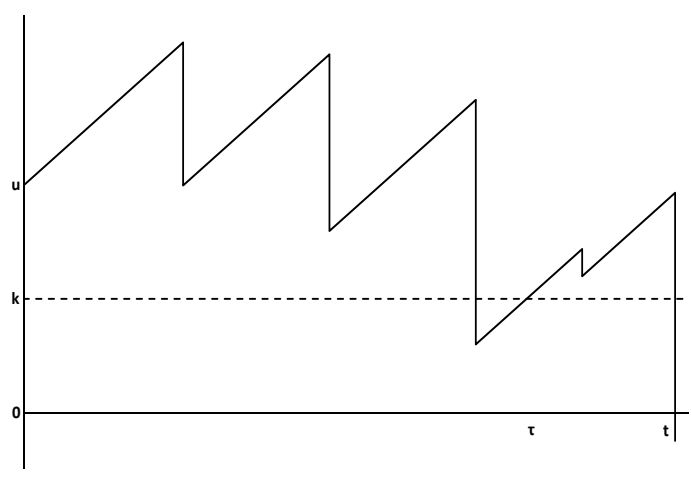

Figure 5.8: Classical surplus process uncrosses $k$ at $\tau$; ruin at $t$ without capital injections between $\tau$ and $t$

From these figures it is not difficult to see that there exists a version of Prabhu's

15 
formula for the risk model with capital injections. Defining $\varphi_{k}(u, t)=\operatorname{Pr}\left(T_{k}>t \mid U(0)=\right.$ $u$ ) to be the finite time survival probability we have

$$
\begin{aligned}
\varphi_{k}(u, t)= & \mathcal{G}(u+c t-k, t)+\lambda \int_{0}^{t} \varphi_{k}(k, t-\tau)\left[e^{-\lambda \tau}(\bar{F}(u+c \tau-k)-\bar{F}(u+c \tau))\right. \\
& \left.+\int_{0}^{u+c \tau-k} g(x, \tau)(\bar{F}(u+c \tau-x-k)-\bar{F}(u+c \tau-x)) d x\right] d \tau \\
& -c \int_{0}^{t} \varphi_{k}(k, t-\tau) g(u+c \tau-k, \tau) d \tau,
\end{aligned}
$$

which reduces to Prabhu's formula when $k=0$. We remark that this formula can be obtained by establishing a partial integro-differential equation for $\varphi_{k}(u, t)$.

Given the nature of the surplus process with capital injections, where the full amount of claims may not be paid by the insurer, it is rather remarkable that formulae for $w_{k}(u, t)$ and $\varphi_{k}(u, t)$ exist in terms of $\mathcal{G}$ and $g$.

\subsection{Exponentially distributed claims}

We now consider the case when $\bar{F}(x)=e^{-\alpha x}$, where $x \geq 0$ with $\alpha>0$, and obtain three main results. We first find an expression for the joint density of $T_{k}$ and $N_{T_{k}}$ when the initial surplus is $k$. We next show that formula (5.14) leads to a more concise expression for $w_{k}(u, t)$ for $u>k$ than that obtained by Nie et al. (2015), then we show that the formulae are equivalent. The derivation of this equivalence is of interest not just for the risk model with capital injections, but also for the classical risk model as in the case $k=0$ Nie et al.'s (2015) formula for $w_{k}(u, t)$ reduces to the expression for $w(u, t)$ that can be found in Drekic and Willmot (2003), whilst our new expression reduces to the formula for $w(u, t)$ first given in Dickson et al. (2005). Our approach is to adapt procedures in Dickson (2007).

Result 5.1 When the initial surplus is $k$, for $n=1,2,3, \ldots$,

$$
w_{k}(k, n+1, t)=\lambda^{n+1} e^{-\alpha k-(\lambda+\alpha c) t} \sum_{j=0}^{n} \frac{(\alpha c)^{n-j} t^{2 n-j}}{(n-j) !}\left(1-e^{-\alpha k}\right)^{j} \frac{j+1}{(n+1) !}
$$

and $w_{k}(k, 1, t)=\lambda e^{-\alpha k-(\lambda+\alpha c) t}$.

Derivation 5.1 We apply formula (5.2). For this we require $\bar{F}_{m, n}(x)$, which can be obtained from (5.3) with $A(x)=e^{-\alpha x}$ and $B_{k}(x)=e^{-\alpha(x+k)}$. Using Laplace transforms we find that

$$
\bar{F}_{m, n}(u)=\frac{e^{-\alpha k(m+1)} e^{-\alpha u} u^{n-1}}{\Gamma(n)} .
$$

Then formula (5.2) yields

$$
\begin{aligned}
w_{k}(k, n+1, t)= & \lambda^{n+1} e^{-\alpha k-(\lambda+\alpha c) t}\left(\left(1-e^{-\alpha k}\right)^{n} \frac{t^{n}}{n !}\right. \\
& \left.+\sum_{j=0}^{n-1} \frac{t^{n-j-1} \alpha^{n-j}}{c^{j+1}(n-j) !}\left(1-e^{-\alpha k}\right)^{j} \int_{0}^{c t} \frac{y^{j+1}(c t-y)^{n-j-1}}{(n-j-1) ! j !} d y\right) .
\end{aligned}
$$


As

$$
\int_{0}^{c t} y^{j+1}(c t-y)^{n-j-1} d y=(c t)^{n+1} \frac{(j+1) !(n-j-1) !}{(n+1) !}
$$

we obtain

$$
w_{k}(k, n+1, t)=\lambda^{n+1} e^{-\alpha k-(\lambda+\alpha c) t} \sum_{j=0}^{n} \frac{(\alpha c)^{n-j} t^{2 n-j}}{(n-j) !}\left(1-e^{-\alpha k}\right)^{j} \frac{j+1}{(n+1) !} .
$$

The formula for $w_{k}(k, 1, t)$ follows immediately from formula (5.6).

Further, we can find $w_{k}(k, t)$ by summing $w_{k}(k, n, t)$, and if we do this we obtain

$$
w_{k}(k, t)=\lambda e^{-\alpha k-(\lambda+\alpha c) t} \sum_{n=1}^{\infty} \frac{\left(\lambda t\left(1-e^{-\alpha k}\right)\right)^{n-1}}{(n-1) !}{ }_{0} F_{1}\left(n+1 ; \lambda \alpha c t^{2}\right)
$$

where ${ }_{0} F_{1}(b ; x)=\sum_{n=0}^{\infty}\left(\Gamma(b) x^{n}\right) /(\Gamma(b+n) n$ !) is a hypergeometric function. We remark that formula (5.15) was obtained by Nie et al. (2015) using a different approach.

Result 5.2 When $u>k$,

$$
\begin{aligned}
w_{k}(u, t)= & \lambda e^{-\alpha u-(\lambda+\alpha c) t} \sum_{n=0}^{\infty} \frac{\left(1-e^{-\alpha k}\right)^{n} \lambda^{n} t^{n / 2}}{(\alpha \lambda(u+c t-k))^{n / 2}} \\
& \times\left(I_{n}(\sqrt{4 \alpha \lambda t(u+c t-k)})-\frac{c t}{u+c t-k} I_{n+2}(\sqrt{4 \alpha \lambda t(u+c t-k)})\right)
\end{aligned}
$$

where

$$
I_{\nu}(t)=\sum_{n=0}^{\infty} \frac{(t / 2)^{2 n+\nu}}{n !(n+\nu) !}
$$

is the modified Bessel function of order $\nu$.

Derivation 5.2 The first two terms of equation (5.14) can be written as

$$
\begin{aligned}
& \lambda e^{-\lambda t} \bar{F}(u+c t)+\lambda \int_{0}^{u+c t-k} \bar{F}(u+c t-x) g(x, t) d x \\
= & \lambda e^{-\alpha u-(\lambda+\alpha c) t} I_{0}(\sqrt{4 \alpha \lambda t(u+c t-k)}) .
\end{aligned}
$$

Next we consider the third term of equation (5.14). Inserting for $\bar{F}$ the first term in the bracket gives us

$$
\lambda e^{-\lambda \tau}(\bar{F}(u+c \tau-k)-\bar{F}(u+c \tau))=\lambda e^{-\lambda \tau}\left(1-e^{-\alpha k}\right) e^{-\alpha(u+c \tau-k)},
$$

and the second term in the bracket becomes

$$
\int_{0}^{u+c \tau-k} g(x, \tau) \lambda(\bar{F}(u+c \tau-x-k)-\bar{F}(u+c \tau-x)) d x
$$




$$
=\lambda \sum_{n=1}^{\infty} e^{-\lambda \tau} \frac{(\lambda \tau)^{n}}{n !} \frac{[\alpha(u+c \tau-k)]^{n}}{n !} e^{-\alpha(u+c \tau-k)}\left(1-e^{-\alpha k}\right) .
$$

Further $w_{k}(k, t-\tau)$ comes from formula (5.15). Hence, switching $\tau$ and $t-\tau$, we can write the third term in (5.14) as

$$
\begin{aligned}
& \int_{0}^{t} w_{k}(k, t-\tau)\left[\lambda e^{-\lambda \tau}(\bar{F}(u+c \tau-k)-\bar{F}(u+c \tau))\right. \\
& \left.+\int_{0}^{u+c \tau-k} g(x, \tau) \lambda(\bar{F}(u+c \tau-x-k)-\bar{F}(u+c \tau-x)) d x\right] d \tau \\
= & \lambda \int_{0}^{t} \sum_{r=0}^{\infty} e^{-\lambda(t-\tau)} \frac{(\lambda(t-\tau))^{r}}{r !}\left(1-e^{-\alpha k}\right) \frac{\alpha^{r} e^{-\alpha(u+c(t-\tau)-k)}}{r !}(u+c(t-\tau)-k)^{r} \\
& \times \sum_{n=1}^{\infty}\left(1-e^{-\alpha k}\right)^{n-1} e^{-\alpha k} \lambda^{n} \tau^{n-1} n e^{-\tau(\lambda+\alpha c)} \sum_{m=0}^{\infty} \frac{\left(\alpha c \lambda \tau^{2}\right)^{m}}{m !(n+m) !} d \tau \\
= & \lambda e^{-\alpha u-(\lambda+\alpha c) t} \sum_{n=1}^{\infty}\left(1-e^{-\alpha k}\right)^{n} \lambda^{n} n \int_{0}^{t} \eta_{1}(\tau) \eta_{2}(t-\tau) d \tau,
\end{aligned}
$$

where

$$
\eta_{1}(t)=t^{n-1} \sum_{m=0}^{\infty} \frac{\left(\alpha c \lambda t^{2}\right)^{m}}{m !(n+m) !}=\frac{t^{-1}}{(\alpha c \lambda)^{n / 2}} I_{n}\left(\sqrt{4 \alpha c \lambda t^{2}}\right)
$$

and

$$
\eta_{2}(t)=\sum_{n=0}^{\infty} \frac{[\alpha \lambda t(u+c t-k)]^{n}}{n ! n !}=I_{0}\left((4 \alpha \lambda c)^{1 / 2} \sqrt{t^{2}+\frac{u-k}{c} t}\right) .
$$

We need two auxiliarly results, the first of which was used in Dickson (2007). First, from Erdélyi (1954, page 201) we have that if

$$
\phi(t)=\frac{t^{\nu / 2}}{(t+\beta)^{\nu / 2}} I_{\nu}\left(A \sqrt{t^{2}+\beta t}\right)
$$

then

$$
\tilde{\phi}(s)=\frac{A^{\nu}}{\sqrt{s^{2}-A^{2}}} \frac{1}{\left(s+\sqrt{s^{2}-A^{2}}\right)^{\nu}} \exp \left\{\frac{\beta}{2}\left(s-\sqrt{s^{2}-A^{2}}\right)\right\} .
$$

Second, from Gradshteyn and Ryzhnik (2007, page 1117), we have that if

$$
\varphi(t)=t^{-1} I_{\nu}(A t)
$$

then

$$
\tilde{\varphi}(s)=\frac{A^{\nu}}{\nu}\left(s+\sqrt{s^{2}-A^{2}}\right)^{-\nu} .
$$

Applying these results with $A=\sqrt{4 \alpha \lambda c}$ and $\beta=(u-k) / c$, we find

$$
\tilde{\eta}_{1}(s) \tilde{\eta}_{2}(s)=\frac{1}{\sqrt{s^{2}-4 \alpha c \lambda}} \exp \left\{\frac{u-k}{2 c}\left(s-\sqrt{s^{2}-4 \alpha \lambda c}\right)\right\} \frac{2^{n}}{n}\left(s+\sqrt{s^{2}-4 \alpha \lambda c}\right)^{-n},
$$


and using formula (5.17) this can be inverted to

$$
\int_{0}^{t} \eta_{1}(\tau) \eta_{2}(t-\tau) d \tau=\frac{(\alpha \lambda c)^{-n / 2}}{n} \frac{t^{n / 2}}{\left(t+\frac{u-k}{c}\right)^{n / 2}} I_{n}(\sqrt{4 \alpha \lambda t(u+c t-k)}) .
$$

The last term in equation (5.14) can be evaluated as

$$
\begin{aligned}
& -c \int_{0}^{t} w_{k}(k, t-\tau) g(u+c \tau-k, \tau) d \tau \\
= & -c \int_{0}^{t} \sum_{r=1}^{\infty} e^{-\lambda(t-\tau)} \frac{(\lambda(t-\tau))^{r}}{r !} \frac{\alpha^{r} e^{-\alpha(u+c(t-\tau)-k)}}{(r-1) !}(u+c(t-\tau)-k)^{r-1} \\
& \times \sum_{n=1}^{\infty}\left(1-e^{-\alpha k}\right)^{n-1} e^{-\alpha k} \lambda^{n} n \tau^{n-1} e^{-\tau(\lambda+\alpha c)} \sum_{m=0}^{\infty} \frac{\left(\alpha c \lambda \tau^{2}\right)^{m}}{m !(n+m) !} d \tau \\
= & -c e^{-\alpha u-(\lambda+\alpha c) t} \sum_{n=1}^{\infty}\left(1-e^{-\alpha k}\right)^{n-1} \lambda^{n} n \int_{0}^{t} \eta_{1}(\tau) \eta_{3}(t-\tau) d \tau,
\end{aligned}
$$

where $\eta_{3}$ is given by

$$
\begin{aligned}
\eta_{3}(t) & =\frac{\alpha \lambda t}{\sqrt{\alpha \lambda t(u+c t-k)}} I_{1}(\sqrt{4 \alpha \lambda t(u+c t-k)}) \\
& =\frac{\sqrt{\alpha \lambda t / c}}{\sqrt{\frac{u-k}{c}+t}} I_{1}\left(\sqrt{4 \alpha \lambda c} \sqrt{\frac{u-k}{c} t+t^{2}}\right)
\end{aligned}
$$

and by (5.17) the Laplace transform of $\eta_{3}$ is found as

$$
\tilde{\eta}_{3}(s)=\frac{\sqrt{\alpha \lambda / c} \sqrt{4 \alpha \lambda c}}{\sqrt{s^{2}-4 \alpha \lambda c}} \frac{1}{s+\sqrt{s^{2}-4 \alpha \lambda c}} \exp \left\{\frac{u-k}{2 c}\left(s-\sqrt{s^{2}-4 \alpha \lambda c}\right)\right\} .
$$

Therefore $\tilde{\eta}_{1}(s) \tilde{\eta}_{3}(s)$ inverts to

$$
\int_{0}^{t} \eta_{1}(\tau) \eta_{3}(t-\tau) d \tau=\frac{\alpha \lambda}{n(\sqrt{\alpha \lambda c})^{n+1}}\left(\frac{t}{t+\frac{u-k}{c}}\right)^{(n+1) / 2} I_{n+1}(\sqrt{4 \alpha \lambda t(u+c t-k)}) .
$$

As a result we can use simple algebra to write equation (5.14) as

$$
\begin{aligned}
w_{k}(u, t)= & \lambda e^{-\alpha u-(\lambda+\alpha c) t} \sum_{n=0}^{\infty} \frac{\left(1-e^{-\alpha k}\right)^{n} \lambda^{n} t^{n / 2}}{(\alpha \lambda(u+c t-k))^{n / 2}} \\
& \times\left(I_{n}(\sqrt{4 \alpha \lambda t(u+c t-k)})-\frac{c t}{u+c t-k} I_{n+2}(\sqrt{4 \alpha \lambda t(u+c t-k)})\right) .
\end{aligned}
$$

Setting $k=0$ in formula (5.16) yields formula (3.9) in Dickson et al. (2005) for the density of the time of ruin in the classical risk model.

Formula (5.16) is a simpler expression than that given for $w_{k}(u, t)$ by Nie et al. (2015) as it is based on infinite sums of Bessel functions, whereas the formula given by Nie et al. (2015) involves double infinite summation of Bessel functions. We now show how formula (5.16) can be manipulated to obtain the expression given in Nie et al. (2015). 
Result 5.3 We can express formula (5.16) as

$$
\begin{aligned}
w_{k}(u, t)= & e^{-\alpha u-(\lambda+\alpha c) t} \sum_{n=0}^{\infty}\left(1-e^{-\alpha k}\right)^{n} \sum_{j=1}^{\infty} \frac{[\alpha(u-k)]^{j-1}}{\Gamma(j)}(\lambda t)^{n+j} t^{-1} \\
& \times \frac{1}{\Gamma(n+j)}{ }_{0} F_{1}\left(n+j+1 ; \alpha \lambda c t^{2}\right) .
\end{aligned}
$$

Derivation 5.3 Expanding the modified Bessel functions in formula (5.16) yields

$$
\begin{aligned}
w_{k}(u, t)= & \lambda e^{-\alpha u-(\lambda+\alpha c) t} \sum_{n=0}^{\infty}\left(1-e^{-\alpha k}\right)^{n}(\lambda t)^{n} \sum_{m=0}^{\infty} \frac{[\alpha \lambda t(u+c t-k)]^{m}}{m !(n+m) !} \\
& -c e^{-\alpha u-(\lambda+\alpha c) t} \sum_{n=0}^{\infty}\left(1-e^{-\alpha k}\right)^{n}(\lambda t)^{n+2} \alpha \sum_{m=0}^{\infty} \frac{[\alpha \lambda t(u+c t-k)]^{m}}{m !(m+n+2) !}
\end{aligned}
$$

and using a binomial expansion gives

$$
\begin{aligned}
& w_{k}(u, t) \\
& =\lambda e^{-\alpha u-(\lambda+\alpha c) t} \sum_{n=0}^{\infty}\left(1-e^{-\alpha k}\right)^{n}(\lambda t)^{n} \sum_{m=0}^{\infty} \frac{(\alpha \lambda t)^{m}}{m !(n+m) !} \sum_{j=0}^{m}\left(\begin{array}{c}
m \\
j
\end{array}\right)(u-k)^{j}(c t)^{m-j} \\
& -c e^{-\alpha u-(\lambda+\alpha c) t} \sum_{n=0}^{\infty}\left(1-e^{-\alpha k}\right)^{n}(\lambda t)^{n+2} \sum_{m=0}^{\infty} \frac{\alpha(\alpha \lambda t)^{m}}{m !(m+n+2) !} \sum_{j=0}^{m}\left(\begin{array}{c}
m \\
j
\end{array}\right)(u-k)^{j}(c t)^{m-j} .
\end{aligned}
$$

Interchanging the order of the inner summations we find

$$
\begin{aligned}
& w_{k}(u, t) \\
& =\lambda e^{-\alpha u-(\lambda+\alpha c) t} \sum_{n=0}^{\infty}\left(1-e^{-\alpha k}\right)^{n} \lambda^{n} t^{n} \sum_{j=0}^{\infty} \sum_{m=j}^{\infty} \frac{(\alpha \lambda t)^{m}}{m !(n+m) !}\left(\begin{array}{c}
m \\
j
\end{array}\right)(u-k)^{j}(c t)^{m-j} \\
& -c e^{-\alpha u-(\lambda+\alpha c) t} \sum_{n=0}^{\infty}\left(1-e^{-\alpha k}\right)^{n}(\lambda t)^{n+2} \sum_{j=0}^{\infty} \sum_{m=j}^{\infty} \frac{\alpha(\alpha \lambda t)^{m}}{m !(m+n+2) !}\left(\begin{array}{c}
m \\
j
\end{array}\right)(u-k)^{j}(c t)^{m-j},
\end{aligned}
$$

and changing the variables of summation we obtain

$$
\begin{aligned}
& w_{k}(u, t) \\
= & \lambda e^{-\alpha u-(\lambda+\alpha c) t} \sum_{n=0}^{\infty}\left(1-e^{-\alpha k}\right)^{n} \lambda^{n} t^{n} \sum_{j=1}^{\infty} \sum_{m=0}^{\infty} \frac{(\alpha \lambda t)^{m+j-1}(u-k)^{j-1}(c t)^{m}}{m !(j-1) !(n+m+j-1) !} \\
& -c e^{-\alpha u-(\lambda+\alpha c) t} \sum_{n=0}^{\infty}\left(1-e^{-\alpha k}\right)^{n}(\lambda t)^{n+2} \sum_{j=1}^{\infty} \sum_{m=0}^{\infty} \frac{\alpha(\alpha \lambda t)^{m+j-1}(u-k)^{j-1}(c t)^{m}}{m !(j-1) !(n+m+j+1) !} \\
= & e^{-\alpha u-(\lambda+\alpha c) t} \sum_{n=0}^{\infty}\left(1-e^{-\alpha k}\right)^{n} \sum_{j=1}^{\infty} \frac{[\alpha(u-k)]^{j-1}}{\Gamma(j)}(\lambda t)^{n+j} t^{-1} \\
& \times\left\{\sum_{m=0}^{\infty} \frac{\left(\alpha \lambda c t^{2}\right)^{m}}{m !(m+n+j-1) !}-\sum_{m=0}^{\infty} \frac{\left(\alpha \lambda c t^{2}\right)^{m+1}}{m !(m+n+j+1) !}\right\} \\
= & e^{-\alpha u-(\lambda+\alpha c) t} \sum_{n=0}^{\infty}\left(1-e^{-\alpha k}\right)^{n} \sum_{j=1}^{\infty} \frac{[\alpha(u-k)]^{j-1}}{\Gamma(j)}(\lambda t)^{n+j} t^{-1}
\end{aligned}
$$




$$
\times \frac{1}{(t \sqrt{\alpha \lambda c})^{n+j-1}}\left\{I_{n+j-1}(2 t \sqrt{\alpha \lambda c})-I_{n+j+1}(2 t \sqrt{\alpha \lambda c})\right\} .
$$

Application of the identity $I_{\nu-1}(t)-I_{\nu+1}(t)=2 \nu I_{\nu}(t) / t$ (see, e.g., Abramowitz and Stegun (1972, formula 9.6.26)) yields the formula for $w_{k}(u, t)$ in Nie et al. (2015), namely

$$
\begin{aligned}
w_{k}(u, t)= & e^{-\alpha u-(\lambda+\alpha c) t} \sum_{n=0}^{\infty}\left(1-e^{-\alpha k}\right)^{n} \sum_{j=1}^{\infty} \frac{[\alpha(u-k)]^{j-1}}{\Gamma(j)}(\lambda t)^{n+j} t^{-1} \\
& \times \frac{n+j}{(t \sqrt{\alpha \lambda c})^{n+j}} I_{n+j}(2 t \sqrt{\alpha \lambda c}) \\
= & e^{-\alpha u-(\lambda+\alpha c) t} \sum_{n=0}^{\infty}\left(1-e^{-\alpha k}\right)^{n} \sum_{j=1}^{\infty} \frac{[\alpha(u-k)]^{j-1}}{\Gamma(j)}(\lambda t)^{n+j} t^{-1} \\
& \times \frac{1}{\Gamma(n+j)}{ }_{0} F_{1}\left(n+j+1 ; \alpha \lambda c t^{2}\right)
\end{aligned}
$$

where we have used the identity

$$
I_{\nu}(z)=\frac{1}{\Gamma(\nu+1)}\left(\frac{z}{2}\right)^{\nu}{ }_{0} F_{1}\left(\nu+1 ; z^{2} / 4\right) .
$$

Setting $k=0$ in formula (5.18) gives formula (2.7) of Drekic and Willmot (2003). Thus, our technique also establishes the identity of their formula with the comparatively simpler formula for $w(u, t)$ given in Dickson et al. (2005).

\subsection{Discussion}

Unfortunately it appears to be difficult to obtain relatively simple expressions for $w_{k}(u, t)$ for other individual claim amount distributions. However, by using numerical integration it should be possible to obtain values for $w_{k}(u, t)$ using formula (5.14). For example, Willmot (2015) gives an expression for $g(x, t)$ when the individual claim amount distribution is an infinite mixture of Erlangs. Alternatively, we might approximate $g(x, t)$ using Panjer's (1981) recursion formula, or we could use approximation techniques discussed by Seal (1978) who considers finite time non-ruin probabilities in the classical risk model.

Applying techniques in Albrecher and Boxma (2005) we can obtain moments of $T_{k}$ and $N_{T_{k}}$ as well as quantities like $\operatorname{Cov}\left(T_{k}, N_{T_{k}}\right)$. Details are relatively straightforward and so we have omitted them. We conducted a number of experiments in calculating the correlation coefficient between $T_{k}$ and $N_{T_{k}}$ when the individual claim amount distribution is exponential. We observed that for a range of values of $u$ and $k$ and different premium loading factors the correlation coefficient was (unsurprisingly) very close to 1 in all our scenarios.

\section{An alternative approach}

We now consider an alternative representation of the Gerber-Shiu function. Let $w_{k}(u, x, y, n, t)$ denote the joint density of the surplus prior to ruin $(x)$, the deficit at ruin $(y)$, the 
number of claims until ruin $(n)$, and the time of ruin $(t)$, with corresponding notation $w(u, x, y, n, t)$ for the classical risk model. Further let

$$
w(u, y, n, t)=\int_{0}^{\infty} w(u, x, y, n, t) d x .
$$

Then

$$
\begin{aligned}
m_{r, \delta}(u)= & E\left[r^{N_{T_{k}}} e^{-\delta T_{k}} \omega\left(U\left(T_{k}^{-}\right),\left|U\left(T_{k}\right)\right|\right) I\left(T_{k}<\infty\right) \mid U(0)=u\right] \\
= & \sum_{n=1}^{\infty} r^{n} \int_{0}^{\infty} e^{-\delta t} \int_{k}^{\infty} \int_{0}^{\infty} \omega(x, y) w_{k}(u, x, y, n, t) d y d x d t \\
= & \sum_{n=1}^{\infty} r^{n} \int_{0}^{\infty} e^{-\delta t}\left\{\int_{0}^{\infty} \int_{0}^{k} w(u-k, x, y, n, t) m_{r, \delta}(k) d y d x\right. \\
& \left.+\int_{0}^{\infty} \int_{k}^{\infty} \omega(x+k, y-k) w(u-k, x, y, n, t) d y d x\right\} d t
\end{aligned}
$$

From this expression we now derive an expression for the probability function of the number of claims until ruin when claims are exponentially distributed, and we show how this expression leads to an expression for the ultimate ruin probability $\psi_{k}(u)$. Our results both generalise and simplify some existing results for the classical risk model.

If we set $\delta>0,0<r<1$ and $\omega(x, y)=1$ for all $x$ and $y$ in equations (6.1) and (6.2), they respectively become

$$
\begin{aligned}
m_{r, \delta}(u) & =\sum_{n=1}^{\infty} r^{n} \int_{0}^{\infty} e^{-\delta t} \int_{k}^{\infty} \int_{0}^{\infty} w_{k}(u, x, y, n, t) d y d x d t \\
& =\sum_{n=1}^{\infty} r^{n} \int_{0}^{\infty} e^{-\delta t} w_{k}(u, n, t) d t=\sum_{n=1}^{\infty} r^{n} w_{k, \delta}(u, n)
\end{aligned}
$$

where $w_{k, \delta}(u, n)=\int_{0}^{\infty} e^{-\delta t} w_{k}(u, n, t) d t$ (with $w_{\delta}(u, n)$ similarly defined), and

$$
\begin{aligned}
m_{r, \delta}(u)= & \sum_{n=1}^{\infty} r^{n} \int_{0}^{\infty} e^{-\delta t} \int_{0}^{\infty} \int_{0}^{k} w(u-k, x, y, n, t) m_{r, \delta}(k) d y d x d t \\
& +\sum_{n=1}^{\infty} r^{n} \int_{0}^{\infty} e^{-\delta t} \int_{0}^{\infty} \int_{k}^{\infty} w(u-k, x, y, n, t) d y d x d t
\end{aligned}
$$

Defining $w_{\delta}(u, y, n)=\int_{0}^{\infty} e^{-\delta t} w(u, y, n, t) d t$ and $\mu_{r, \delta}(u, y)=\sum_{n=1}^{\infty} r^{n} w_{\delta}(u, y, n)$ we get

$$
m_{r, \delta}(u)=m_{r, \delta}(k) \int_{0}^{k} \mu_{r, \delta}(u-k, y) d y+\int_{k}^{\infty} \mu_{r, \delta}(u-k, y) d y .
$$

In particular, when $u=k$ we have

$$
m_{r, \delta}(k)=\frac{\int_{k}^{\infty} \mu_{r, \delta}(0, y) d y}{1-\int_{0}^{k} \mu_{r, \delta}(0, y) d y}
$$

For the remainder of this section we consider the case $\bar{F}(x)=e^{-\alpha x}$ for $x \geq 0$ with $\alpha>0$, and let $p_{k}(u, n)=\operatorname{Pr}\left(N_{T_{k}}=n \mid U(0)=u\right)$ for $n=1,2,3, \ldots$ denote the probability function of the number of claims until ruin. 
Result 6.1 When the initial surplus is $k$, for $n=1,2,3, \ldots$

$$
p_{k}(k, n)=e^{-\alpha k} \sum_{j=0}^{n-1}\left(1-e^{-\alpha k}\right)^{j}\left(\frac{\alpha c}{\lambda+\alpha c}\right)^{n-j-1}\left(\frac{\lambda}{\lambda+\alpha c}\right)^{n} \frac{(j+1)(2 n-j-2) !}{n !(n-j-1) !} .
$$

Derivation 6.1 It is easy to show from results for the classical risk model in Dickson (2012) that $w(u, y, n, t)=w(u, n, t) \alpha e^{-\alpha y}$, which gives $\mu_{r, \delta}(u, y)=\mu_{r, \delta}(u) \alpha e^{-\alpha y}$ where $\mu_{r, \delta}(u)=\int_{0}^{\infty} \mu_{r, \delta}(u, y) d y$. Hence formula (6.5) can be written as

$$
m_{r, \delta}(k)=\frac{\mu_{r, \delta}(0) e^{-\alpha k}}{1-\mu_{r, \delta}(0)\left(1-e^{-\alpha k}\right)}=\sum_{i=0}^{\infty} e^{-\alpha k}\left(1-e^{-\alpha k}\right)^{i} \mu_{r, \delta}(0)^{i+1},
$$

where for $i=0,1,2, \ldots$

$$
\mu_{r, \delta}(0)^{i+1}=\sum_{n=i+1}^{\infty} r^{n} \int_{0}^{\infty} e^{-\delta t} w^{(i+1) *}(0, n, t) d t
$$

and for $i=1,2,3, \ldots$

$$
w^{(i+1) *}(0, n, t)=\sum_{j=1}^{n-1} \int_{0}^{t} w^{i *}(0, j, s) w(0, n-j, t-s) d s .
$$

Inverting formula (6.7) with respect to $\delta$ yields

$$
\sum_{n=1}^{\infty} r^{n} w_{k}(k, n, t)=\sum_{i=0}^{\infty} e^{-\alpha k}\left(1-e^{-\alpha k}\right)^{i} \sum_{n=i+1}^{\infty} r^{n} w^{(i+1) *}(0, n, t) .
$$

Applying the techniques in Nie et al. (2015) we can show that

$$
\sum_{n=i+1}^{\infty} r^{n} w^{(i+1) *}(0, n, t)=(r \lambda)^{i+1} e^{-(\lambda+\alpha c) t} t^{i}(i+1) \sum_{n=0}^{\infty} \frac{\left(r \alpha c \lambda t^{2}\right)^{n}}{n !(n+i+1) !},
$$

and inserting this in equation (6.8) we obtain

$$
\sum_{n=1}^{\infty} r^{n} w_{k}(k, n, t)=\sum_{n=1}^{\infty}(r \lambda)^{n} e^{-\alpha k}\left(1-e^{-\alpha k}\right)^{n-1} e^{-(\lambda+\alpha c) t} t^{n-1} n \sum_{j=0}^{\infty} \frac{\left(\alpha r c \lambda t^{2}\right)^{j}}{j !(j+n) !} .
$$

Integration over $t$ yields the probability generating function of $N_{T_{k}}$ given $u=k$, denoted by $\tilde{p}_{k, r}(k)$, as

$$
\tilde{p}_{k, r}(k)=e^{-\alpha k} \sum_{n=1}^{\infty}(r \lambda)^{n}\left(1-e^{-\alpha k}\right)^{n-1} n \sum_{j=0}^{\infty} \frac{\Gamma(2 j+n)}{j ! \Gamma(n+1+j)} \frac{(\alpha r c \lambda)^{j}}{(\lambda+\alpha c)^{2 j+n}},
$$

and applying the identity $\sum_{n=1}^{\infty} \sum_{j=0}^{\infty} t_{n, j}=\sum_{n=1}^{\infty} \sum_{j=0}^{n-1} t_{j+1, n-j-1}$ we obtain

$$
\tilde{p}_{k, r}(k)=e^{-\alpha k} \sum_{n=1}^{\infty}(r \lambda)^{n} \sum_{j=0}^{n-1}\left(1-e^{-\alpha k}\right)^{j} \frac{(j+1) \Gamma(2 n-j-1)}{(n-j-1) ! \Gamma(n+1)} \frac{(\alpha c)^{n-j-1}}{(\lambda+\alpha c)^{2 n-j-1}},
$$

from which the result follows. 
Setting $k=0$ in formula (6.6) yields the probability function of the number of claims until ruin from initial surplus 0 in the classical risk model; see, for example, Landriault et al. (2011).

Result 6.2 When $u>k$, for $n=1,2,3, \ldots$

$$
\begin{aligned}
p_{k}(u, n)= & e^{-\alpha u} \sum_{i=0}^{n-1} \sum_{j=0}^{i}\left(1-e^{-\alpha k}\right)^{j} \frac{(\alpha(u-k))^{i-j}}{(i-j) !}\left(\frac{\alpha c}{\lambda+\alpha c}\right)^{n-i-1}\left(\frac{\lambda}{\lambda+\alpha c}\right)^{n} \\
& \times \frac{(i+1)(2 n-i-2) !}{n !(n-i-1) !}
\end{aligned}
$$

Derivation 6.2 Formula (6.4) can be written as

$$
\begin{aligned}
m_{r, \delta}(u) & =m_{r, \delta}(k) \mu_{r, \delta}(u-k)\left(1-e^{-\alpha k}\right)+\mu_{r, \delta}(u-k) e^{-\alpha k} \\
& =\sum_{i=0}^{\infty} e^{-\alpha k}\left(1-e^{-\alpha k}\right)^{i} \mu_{r, \delta}(0)^{i} \mu_{r, \delta}(u-k) .
\end{aligned}
$$

To apply this expression we need an expression for $\mu_{r, \delta}(u)$ in terms of $\mu_{r, \delta}(0)$, and to obtain this we extend the approach in Dickson and Li (2010). We first note that

$$
w(u, 1, t)=\int_{u}^{\infty} w(0, y, 1, t) d y=w(0,1, t) e^{-\alpha u}
$$

and for $n=2,3,4, \ldots$,

$$
\begin{aligned}
w(u, n, t) & =\sum_{j=1}^{n-1} \int_{0}^{t} \int_{0}^{u} w(0, y, j, \tau) w(u-y, n-j, t-\tau) d y d \tau+\int_{u}^{\infty} w(0, y, n, t) d y \\
& =\sum_{j=1}^{n-1} \int_{0}^{t} \int_{0}^{u} w(0, j, \tau) \alpha e^{-\alpha y} w(u-y, n-j, t-\tau) d y d \tau+\int_{u}^{\infty} w(0, n, t) \alpha e^{-\alpha y} d y .
\end{aligned}
$$

Define

$$
\tilde{\mu}_{r, \delta}(s)=\int_{0}^{\infty} e^{-s u} \mu_{r, \delta}(u) d u=\sum_{n=1}^{\infty} r^{n} \int_{0}^{\infty} \int_{0}^{\infty} e^{-s u-\delta t} w(u, n, t) d t d u=\sum_{n=1}^{\infty} r^{n} \tilde{w}_{\delta}(s, n) .
$$

Taking the bivariate Laplace transform of formula (6.12) gives

$$
\tilde{w}_{\delta}(s, 1)=w_{\delta}(0,1) \frac{1}{\alpha+s}
$$

and the Laplace transform of formula (6.13) with respect to $t$ is

$$
w_{\delta}(u, n)=\sum_{j=1}^{n-1} w_{\delta}(0, j) \int_{0}^{u} \alpha e^{-\alpha y} w_{\delta}(u-y, n-j) d y+w_{\delta}(0, n) e^{-\alpha u} .
$$

Now take the Laplace transform of formula (6.14) with respect to $u$, which results in

$$
\tilde{w}_{\delta}(s, n)=\sum_{j=1}^{n-1} w_{\delta}(0, j) \tilde{w}_{\delta}(s, n-j) \frac{\alpha}{\alpha+s}+w_{\delta}(0, n) \frac{1}{\alpha+s} .
$$


Multiplying formula (6.15) by $r^{n}$ and summing over $n$, we have

$$
\sum_{n=2}^{\infty} r^{n} \tilde{w}_{\delta}(s, n)=\sum_{n=2}^{\infty} r^{n} \sum_{j=1}^{n-1} w_{\delta}(0, j) \tilde{w}_{\delta}(s, n-j) \frac{\alpha}{\alpha+s}+\sum_{n=2}^{\infty} r^{n} w_{\delta}(0, n) \frac{1}{\alpha+s} .
$$

Adding $r \tilde{w}_{\delta}(s, 1)$ to both sides of (6.16) gives

$$
\sum_{n=1}^{\infty} r^{n} \tilde{w}_{\delta}(s, n)=\sum_{n=2}^{\infty} r^{n} \sum_{j=1}^{n-1} w_{\delta}(0, j) \tilde{w}_{\delta}(s, n-j) \frac{\alpha}{\alpha+s}+\sum_{n=1}^{\infty} r^{n} w_{\delta}(0, n) \frac{1}{\alpha+s}
$$

which can be written as

$$
\begin{aligned}
\tilde{\mu}_{r, \delta}(s) & =\mu_{r, \delta}(0) \tilde{\mu}_{r, \delta}(s) \frac{\alpha}{\alpha+s}+\mu_{r, \delta}(0) \frac{1}{\alpha+s} \\
& =\frac{\mu_{r, \delta}(0) \frac{1}{\alpha+s}}{1-\mu_{r, \delta}(0) \frac{\alpha}{\alpha+s}}=\frac{1}{\alpha} \sum_{n=1}^{\infty} \mu_{r, \delta}(0)^{n}\left(\frac{\alpha}{\alpha+s}\right)^{n} .
\end{aligned}
$$

Inverting $\tilde{\mu}_{r, \delta}(s)$ with respect to $s$ yields

$$
\mu_{r, \delta}(u)=\sum_{n=1}^{\infty} \mu_{r, \delta}(0)^{n} \frac{(\alpha u)^{n-1} e^{-\alpha u}}{(n-1) !}
$$

and applying this in equation (6.11) we get

$$
m_{r, \delta}(u)=\sum_{i=0}^{\infty} e^{-\alpha u}\left(1-e^{-\alpha k}\right)^{i} \sum_{n=1}^{\infty} \mu_{r, \delta}(0)^{i+n} \frac{[\alpha(u-k)]^{n-1}}{(n-1) !}
$$

which by (6.9) inverts with respect to $\delta$ to

$$
\sum_{i=0}^{\infty} e^{-\alpha u}\left(1-e^{-\alpha k}\right)^{i} \sum_{n=1}^{\infty}(r \lambda)^{i+n} e^{-(\lambda+\alpha c) t} t^{i+n-1}(i+n) \sum_{j=0}^{\infty} \frac{\left(r \alpha c \lambda t^{2}\right)^{j}}{j !(j+n+i) !} \frac{[\alpha(u-k)]^{n-1}}{(n-1) !} .
$$

Integrating with respect to $t$ we find the probability generating function of $N_{T_{k}}$ as

$$
\begin{aligned}
\tilde{p}_{k, r}(u)= & \sum_{i=0}^{\infty} e^{-\alpha u}\left(1-e^{-\alpha k}\right)^{i} \sum_{n=0}^{\infty}(r \lambda)^{i+n+1} \sum_{j=0}^{\infty} \frac{(r \alpha c \lambda)^{j}(i+n+1)}{j !(j+n+i+1) !} \frac{[\alpha(u-k)]^{n}}{n !} \\
& \times \frac{(2 j+i+n) !}{(\lambda+\alpha c)^{2 j+i+n+1}} .
\end{aligned}
$$

We can then apply the identity

$$
\sum_{i=0}^{\infty} \sum_{n=0}^{\infty} \sum_{j=0}^{\infty} t_{i, n, j}=\sum_{i=0}^{\infty} \sum_{n=0}^{i} \sum_{j=0}^{n} t_{j, n-j, i-n}
$$

(see, for example, Graham et al. (1994, p.355)) giving

$$
\tilde{p}_{k, r}(u)=\sum_{i=0}^{\infty} r^{i+1} e^{-\alpha u} \sum_{n=0}^{i} \sum_{j=0}^{n}\left(1-e^{-\alpha k}\right)^{j} \frac{(\alpha(u-k))^{n-j}}{(n-j) !}\left(\frac{\alpha c}{\lambda+\alpha c}\right)^{i-n}\left(\frac{\lambda}{\lambda+\alpha c}\right)^{i+1}
$$




$$
\times \frac{(n+1)(2 i-n) !}{(i-n) !(i+1) !} .
$$

It follows that $p_{k}(u, 1)=\lambda e^{-\alpha u} /(\lambda+\alpha c)$, and for $n=2,3,4, \ldots$,

$$
\begin{aligned}
p_{k}(u, n)= & e^{-\alpha u} \sum_{i=0}^{n-1} \sum_{j=0}^{i}\left(1-e^{-\alpha k}\right)^{j} \frac{(\alpha(u-k))^{i-j}}{(i-j) !}\left(\frac{\alpha c}{\lambda+\alpha c}\right)^{n-i-1}\left(\frac{\lambda}{\lambda+\alpha c}\right)^{n} \\
& \times \frac{(i+1)(2 n-i-2) !}{n !(n-i-1) !}
\end{aligned}
$$

which also holds for $n=1$.

We remark that setting $u=k$ in formula (6.17) yields formula (6.6). Further, setting $k=0$ in formula $(6.17)$ gives $p(u, n)$, the probability function of the number of claims until ruin in the classical model, as

$$
p(u, n)=e^{-\alpha u} \sum_{i=0}^{n-1} \frac{(\alpha u)^{i}}{i !}\left(\frac{\alpha c}{\lambda+\alpha c}\right)^{n-i-1}\left(\frac{\lambda}{\lambda+\alpha c}\right)^{n} \frac{(i+1)(2 n-i-2) !}{n !(n-i-1) !} .
$$

We remark that this formula is new, and can also be obtained by manipulation of formula (23) of Landriault et al. (2011). We end by obtaining an expression for $\psi_{k}(u)$ by summing formula (6.17).

Result 6.3 For $u \geq k$, the ultimate ruin probability is

$$
\psi_{k}(u)=\frac{\lambda}{\alpha c} \frac{e^{-(\alpha-\lambda / c)(u-k)} e^{-\alpha k}}{1-(\lambda /(\alpha c))\left(1-e^{-\alpha k}\right)} .
$$

Derivation 6.3 We have

$$
\begin{aligned}
& \psi_{k}(u)=\sum_{n=1}^{\infty} p_{k}(u, n) \\
& =e^{-\alpha u} \sum_{n=1}^{\infty} \sum_{i=0}^{n-1} \sum_{j=0}^{i}\left(1-e^{-\alpha k}\right)^{j} \frac{(\alpha(u-k))^{i-j}}{(i-j) !}\left(\frac{\alpha c}{\lambda+\alpha c}\right)^{n-i-1}\left(\frac{\lambda}{\lambda+\alpha c}\right)^{n} \\
& \times \frac{(i+1)(2 n-i-2) !}{n !(n-i-1) !} \\
& =e^{-\alpha u} \sum_{i=0}^{\infty} \sum_{n=i+1}^{\infty} \sum_{j=0}^{i}\left(1-e^{-\alpha k}\right)^{j} \frac{(\alpha(u-k))^{i-j}}{(i-j) !}\left(\frac{\alpha c}{\lambda+\alpha c}\right)^{n-i-1}\left(\frac{\lambda}{\lambda+\alpha c}\right)^{n} \\
& \times \frac{i+1}{2 n-i-1}\left(\begin{array}{c}
2 n-i-1 \\
n
\end{array}\right) \\
& =e^{-\alpha u} \sum_{i=0}^{\infty} \sum_{t=0}^{\infty} \sum_{j=0}^{i}\left(1-e^{-\alpha k}\right)^{j} \frac{(\alpha(u-k))^{i-j}}{(i-j) !}\left(\frac{\alpha c}{\lambda+\alpha c}\right)^{t}\left(\frac{\lambda}{\lambda+\alpha c}\right)^{t+i+1} \\
& \times \frac{i+1}{2 t+i+1}\left(\begin{array}{c}
2 t+i+1 \\
t
\end{array}\right)
\end{aligned}
$$




$$
\begin{aligned}
= & e^{-\alpha u} \sum_{i=0}^{\infty} \sum_{j=0}^{i}\left(1-e^{-\alpha k}\right)^{j} \frac{(\alpha(u-k))^{i-j}}{(i-j) !}\left(\frac{\lambda}{\lambda+\alpha c}\right)^{i+1} \\
& \times \sum_{t=0}^{\infty}\left(\begin{array}{c}
2 t+i+1 \\
t
\end{array}\right) \frac{i+1}{2 t+i+1}\left(\frac{\lambda \alpha c}{(\lambda+\alpha c)^{2}}\right)^{t} \\
= & e^{-\alpha u} \sum_{i=0}^{\infty} \sum_{j=0}^{i}\left(1-e^{-\alpha k}\right)^{j} \frac{(\alpha(u-k))^{i-j}}{(i-j) !}\left(\frac{\lambda}{\lambda+\alpha c}\right)^{i+1} \mathcal{B}_{2}\left(\frac{\lambda \alpha c}{(\lambda+\alpha c)^{2}}\right)^{i+1}
\end{aligned}
$$

where $\mathcal{B}_{2}$ is the generalised binomial series given by

$$
\mathcal{B}_{2}(z)=\sum_{k=0}^{\infty}\left(\begin{array}{c}
2 k+1 \\
k
\end{array}\right) \frac{z^{k}}{2 k+1}=\frac{1-\sqrt{1-4 z}}{2 z}
$$

with the property that

$$
\mathcal{B}_{2}(z)^{r}=\sum_{k=0}^{\infty}\left(\begin{array}{c}
2 k+r \\
k
\end{array}\right) \frac{r z^{k}}{2 k+r} .
$$

See Graham et al. (1994). It is easily seen that

$$
\mathcal{B}_{2}\left(\frac{\lambda \alpha c}{(\lambda+\alpha c)^{2}}\right)=\frac{\lambda+\alpha c}{\alpha c}
$$

giving

$$
\begin{aligned}
\sum_{n=1}^{\infty} p_{k}(u, n) & =e^{-\alpha u} \sum_{i=0}^{\infty} \sum_{j=0}^{i}\left(1-e^{-\alpha k}\right)^{j} \frac{(\alpha(u-k))^{i-j}}{(i-j) !}\left(\frac{\lambda}{\alpha c}\right)^{i+1} \\
& =e^{-\alpha u} \sum_{j=0}^{\infty}\left(1-e^{-\alpha k}\right)^{j} \sum_{i=j}^{\infty} \frac{(\alpha(u-k))^{i-j}}{(i-j) !}\left(\frac{\lambda}{\alpha c}\right)^{i+1} \\
& =\frac{\lambda}{\alpha c} e^{-\alpha u} \sum_{j=0}^{\infty}\left(1-e^{-\alpha k}\right)^{j}\left(\frac{\lambda}{\alpha c}\right)^{j} \sum_{m=0}^{\infty} \frac{(\lambda(u-k) / c)^{m}}{m !} \\
& =\frac{\lambda}{\alpha c} \frac{e^{-(\alpha-\lambda / c)(u-k)} e^{-\alpha k}}{1-(\lambda /(\alpha c))\left(1-e^{-\alpha k}\right)}
\end{aligned}
$$

From formula (4.6) and results in Gerber et al. (1987) we can easily verify that (6.18) does indeed equal $\psi_{k}(u)$.

\section{Concluding remarks}

Our Gerber-Shiu analysis is not as helpful in dealing with infinite time ruin problems as it is with dealing with finite time ruin problems. For the latter it has led us to the rather surprising conclusion that both the density of the time of ruin and the finite time survival (or ruin) probability for our risk model with capital injections can be expressed in terms of the aggregate claims distribution for the classical risk model.

Our analysis in the case of exponentially distributed individual claims has extended existing results for the classical risk model, and has shown the connection between two 
known formulae for the density of the time of ruin in the classical risk model. Our analysis in Section 6 can be extended to other problems, but it is difficult to obtain neat explicit solutions when we consider individual claim amount distributions other than the exponential distribution.

Acknowledgement The authors are grateful to the referees for constructive comments which improved the quality of this paper; in particular, the proof of Theorem 3.1 was greatly improved by a referee's comments.

\section{References}

[1] Abramowitz, M. and Stegun, I.A. (1965). Handbook of mathematical functions. Dover, New York.

[2] Albrecher, H. and Boxma, O. (2005) On the discounted penalty function in a Markovdependent risk model. Insurance: Mathematics \&6 Economics 37, 650-672.

[3] Dickson, D.C.M. (1998) On a class of renewal risk processes. North American Actuarial Journal, 2, $3,60-73$.

[4] Dickson, D.C.M. (2005) Insurance Risk and Ruin. Cambridge University Press, Cambridge.

[5] Dickson, D.C.M. (2007) Some finite time ruin problems. Annals of Actuarial Science $2,217-232$.

[6] Dickson, D.C.M. (2012) The joint distribution of the time to ruin and the number of claims until ruin in the classical risk model. Insurance: Mathematics $\&$ Economics $50,334-337$.

[7] Dickson, D.C.M. and Hipp, C. (2001) On the time to ruin for Erlang(2) risk processes. Insurance: Mathematics \& Economics 29, 333-344.

[8] Dickson, D.C.M., Hughes, B.D. and Lianzeng, Z. (2005). The density of the time to ruin for a Sparre Andersen process with Erlang arrivals and exponential claims. Scandinavian Actuarial Journal, 2005, 358-376.

[9] Dickson, D.C.M. and Li, S. (2010) Finite time ruin problems for the Erlang(2) risk model. Insurance: Mathematics \& Economics 46, 12-18.

[10] Drekic, S. and Willmot, G.E. (2003). On the density and moments of the time to ruin with exponential claims. ASTIN Bulletin 33, 11-21.

[11] Erdélyi, A. (ed.) (1954). Tables of integral transforms, Volume 1. McGraw-Hill, New York.

[12] Gerber, H.U., Goovaerts, M.J., and Kaas, R. (1987). On the probability and severity of ruin. ASTIN Bulletin 17, 151-163.

[13] Gerber, H. U. and Shiu, E. S. W. (1998). On the time value of ruin. North American Actuarial Journal 2, 1, 48-78. 
[14] Gerber, H. U. and Shiu, E. S. W. (2005). The time value of ruin in a Sparre Andersen model. North American Actuarial Journal 9, 2, 1-21.

[15] Gradshteyn, I.S. and Ryzhnik, I.M. (2007) Table of integrals, series, and products (Seventh edition). Academic Press, San Diego.

[16] Graham, R.L., Knuth, D.E. and Patashnik, O. (1994). Concrete Mathematics, 2nd edition. Addison-Wesley, Upper Saddle River, NJ.

[17] Landriault, D., Shi, T. and Willmot, G.E. (2011). Joint density involving the time to ruin in the Sparre Andersen risk model under exponential assumptions. Insurance: Mathematics \& Economics 49, 371-379.

[18] Li, S. and Lu, Y. (2008) The decompositions of the discounted penalty functions and dividends-penalty identity in a Markov-modulated risk model. ASTIN Bulletin $38,53-71$.

[19] Lin, X.S., Willmot, G.E. and Drekic, S. (2003) The classical Poisson risk model with a constant dividend barrier: analysis of the Gerber-Shiu discounted penalty function. Insurance: Mathematics \&5 Economics, 33, 551-566.

[20] Nie, C., D.C.M. Dickson and S. Li (2011). Minimizing the ruin probability through capital injections. Annals of Actuarial Science 5, 195-209.

[21] Nie, C., D.C.M. Dickson and S. Li (2015) The finite time ruin probability in a risk model with capital injections. Scandinavian Actuarial Journal, 2015, 301-318.

[22] Panjer, H.H. (1981) Recursive evaluation of a family of compound distributions. ASTIN Bulletin 12, 22-26.

[23] Panjer, H.H. and Willmot, G.E. (1992) Insurance Risk Models. Society of Actuaries, Schaumburg, IL.

[24] Prabhu, N.U. (1961) On the ruin problem of collective risk theory. Annals of Mathematical Statistics 32, 757-764.

[25] Seal, H.L. (1978) Survival probabilities - the goal of risk theory. John Wiley 85 Sons, New York.

[26] Willmot, G.E. (2015) On a partial differential equation of Seal's type. Insurance: Mathematics $\&$ Economics 62, 54-61. 


\section{University Library}

\section{- M M I N E R VA A gateway to Melbourne's research publications}

Minerva Access is the Institutional Repository of The University of Melbourne

Author/s:

DICKSON, DCM;Qazvini, M

Title:

Gerber-Shiu analysis of a risk model with capital injections

Date:

2016

Citation:

DICKSON, D. C. M. \& Qazvini, M. (2016). Gerber-Shiu analysis of a risk model with capital injections. European Actuarial Journal, 6 (2), pp.409-440. https://doi.org/10.1007/ s13385-016-0131-1.

Persistent Link:

http://hdl.handle.net/11343/120633 